\title{
RELACIONES FITOGEOGRÁFICAS DE LAS ESPERMATÓFITAS EN LAS PLUVISILVAS DE LA REGIÓN ORIENTAL DE CUBA
}

\section{PHYTOGEOGRAPHIC RELATIONSHIPS OF SPERMATOPHYTES ON THE RAINFOREST OF THE EASTERN REGION OF CUBA}

\author{
Eddy Martínez-Quesada \\ Centro de Investigaciones de Medio Ambiente de Camagüey, Cisneros 105 altos, e/ Pobre y Ángel, \\ Camagüey 1, C.P. 70100, Cuba. \\ eddy@cimac.cu
}

\section{RESUMEN}

Se realizó un estudio de la distribución de las espermatófitas que habitan en las pluvisilvas de la Región Oriental de Cuba, con el objetivo de conocer principalmente sus relaciones con otros sectores y distritos de Cuba así como con otras provincias florísticas de la Región Caribe. Para obtener la distribución de las especies se revisó la bibliografía especializada y en el caso de las especies endémicas se consultó además una base de datos. Cada endemismo se clasificó según el criterio de López et al. (1994a) y se ubicó en las diferentes unidades fitogeográficas de Cuba según Samek (1973). La clasificación de las disyunciones se realizó según el criterio de Borhidi (1991). En el caso de las especies no endémicas se hizo una separación entre los taxones que pertenecen a las pluvisilvas de la Subregión Grupo Orográfico Sagua-Baracoa y la Subregión Sierra Maestra, a partir del criterio de Núñez y Viña (1989). La ubicación de cada taxón en los reinos y regiones del mundo se realizó a partir del criterio de Schmidt (1969). En la Región Caribe se utilizó el de Borhidi y Muñiz (1986), ratificado por Borhidi (1991, 1996). Los centros de origen y evolución de las familias se definieron a partir de Gentry (1982). Para obtener los dendrogramas se confeccionaron tres matrices de presencia y ausencia de especies, una para todos los endemismos y las dos restantes para los no endemismos de la Región Caribe presentes en las pluvisilvas de los subsectores Nor Oriental y Sur Oriental. La clasificación de las pluvisilvas se realizó de acuerdo con el criterio de Reyes \& Acosta (2005), y Reyes (2006). Las especies endémicas se distribuyen en su mayoría en el Sector Cuba Oriental y entre varios distritos del mismo. Fuera de este sector, las relaciones más estrechas son con los distritos Escambray, en Cuba Central y Sierra del Rosario, en Cuba Occidental. Dichas especies endémicas presentan tres tipos de disyunción, pero las que poseen distribución bisectorial y trisectorial son las más numerosas. Las especies no endémicas de las pluvisilvas tienen su mayor afinidad florística con la Región Caribe, Subregión Antillana, provincia La Española, dentro del Neotrópico. Las familias provenientes de Gondwana son predominantes y dentro de éste se destacan las del centro amazónico.

Palabras Clave: Fitogeografía, bosques pluviales, endemismo, Región Oriental de Cuba.

\section{ABSTRACT}

A study about distribution of spermatophytes living on rainforest of Oriental Region of Cuba was performed with the objective to know their relationships to other sectors and districts of Cuba, as well as other floristic provinces from Caribbean Region, mainly. The distribution of species was obtained by bibliographical revision while in those considered endemic species were consulted in addition a data base. Each endemism were classified according to López et al. (1994a) and were located on different phytogeographic units of Cuba according to Samek (1973). The classifications of disjunctions were carried out according to Borhidi (1991). For non-endemic species a separation among taxa belonging rainforest of Grupo Orográfico Sagua-Baracoa Sub-region and those of Sierra Maestra Subregion were done, according to Núñez \& Viña (1989). Each taxon was located in kingdoms and regions of the world according to Schmidt (1969), while in the Caribbean Region were according to Borhidi y Muñiz (1986), ratified by 
Gayana Bot. 66(2), 2009

Borhidi (1991, 1996). Evolutionary centers of families were definite from Gentry (1982). Dendrograms were obtained making tree matrix, one of them for all endemism and the two remainder for non-endemic species of Caribbean Region, which are on rainforest of both Nor Oriental and South Oriental Sub-sector. The classifications of rainforest were carried out according to Reyes \& Acosta (2005) and Reyes (2006). The endemic species are mostly distributed on Cuba Oriental Sector and among several districts in it. In addition to this sector, the nearest relationships are with Escambray district in Central Cuba and secondly to Sierra del Rosario district, in Western Cuba. These endemic species show three types of disjunction, but those that have bisectorial distribution and trisectorial distribution are found to be the greatest. The non-endemic species from rainforest have the nearest relationships to Caribbean Region, Antillean Subregion, La Española province, within Neotropic. Families from Gondwanaland are prevalent and within this, those from Amazonian center are the most important.

KEYwords: Phytogeography, rainforests, endemism, Eastern Region of Cuba.

\section{INTRODUCCION}

Las pluvisilvas son los bosques más exuberantes del planeta. Al mismo tiempo, se considera que en ellos se encuentra el $90 \%$ de la biodiversidad mundial y constituyen los ecosistemas naturales más productivos del mundo (Lastres 1988). En Cuba se presentan en las zonas de mayor pluviosidad y se clasifican de formas diferentes, en dependencia de las disimilitudes geológicas y edáficas del territorio, o de una de éstas (Reyes \& Acosta 2005a, b). En la Región Oriental son las formaciones nemorales más importantes, no sólo por la extensión que ocupan sino también por la riqueza de especies que existe en cada una de ellas, en su mayoría espermatofitas y el alto porcentaje de endemismos que albergan.

La distribución de las especies endémicas en el Sector Cuba Oriental ha sido analizada en distintas ocasiones (Albert \& López 1986, López et al. 1993, 1994a, 1994b, Reyes y del Risco 1994, Fagilde 1998, 2000b, 2001). Respecto a las especies no endémicas, existen en la literatura algunas contribuciones donde se exponen las relaciones fitogeográficas de Cuba con otras partes del Caribe de forma general (Gómez de la Maza \& Roig y Mesa 1914, Alain 1958, Samek 1973), así como de Cuba Oriental en particular (Samek 1973, Martínez 1998, 2000, 2001, Reyes et al. 2000).

Las relaciones fitogeográficas en las formaciones vegetales cubanas, incluso en las del Sector Cuba Oriental, se han abordado poco en la literatura consultada (Samek 1973, Fagilde 2000a). Otros autores que estudiaron la flora y la vegetación en una localidad determinada sólo expusieron los tipos corológicos hallados (García et al. 1985a, 1985b, Ricardo et al. 1985, Capote et al. 1987, Franco et al. 2004). Borhidi (1991) al referirse a los tipos corológicos de Cuba, específicamente a los antillanos, enfatizó que los bosques pluviales y los bosques nublados son ricos en geoelementos de ese tipo.

La fitogeografía de las pluvisilvas no se ha estudiado hasta el presente y aunque se conocen los centros de origen y evolución de las familias en tres tipos de estos bosques (Fagilde 2000a), no se ha intentado establecer las afinidades florísticas de los mismos con los distritos fitogeográficos de Cuba a través de sus especies endémicas, ni tampoco con otras unidades fitogeográficas del mundo, principalmente con el Caribe, mediante las especies no endémicas.

El objetivo del trabajo es realizar un análisis de la distribución de las especies endémicas en Cuba y no endémicas con el resto del mundo, a partir de los diferentes tipos de pluvisilvas y con ello conocer sus relaciones fitogeográficas.

\section{MATERIALES Y METODOS}

RECOLECCIÓN Y COMPILACIÓN DE LOS TAXONES Para este estudio se efectuaron recolectas en 23 localidades de la Región Oriental de Cuba, donde se desarrollan las pluvisilvas estudiadas, aunque el área que ocupan las mismas es mucho más extensa (Fig. 1). También se revisó la literatura y el material depositado en herbarios cubanos donde aparecen las familias, géneros y taxones infragenéricos que se han recolectado en estos tipos de bosques.

\section{ESPECIES ENDÉMICAS}

Los endemismos se definieron a partir de la revisión de la obra Flora de Cuba (León 1946, León, \& Alain 1951, Alain 1953, 1957, 1964, Kuijt 1961, Berazaín 1992, Leiva 1992, Arias 1998, Bässler 


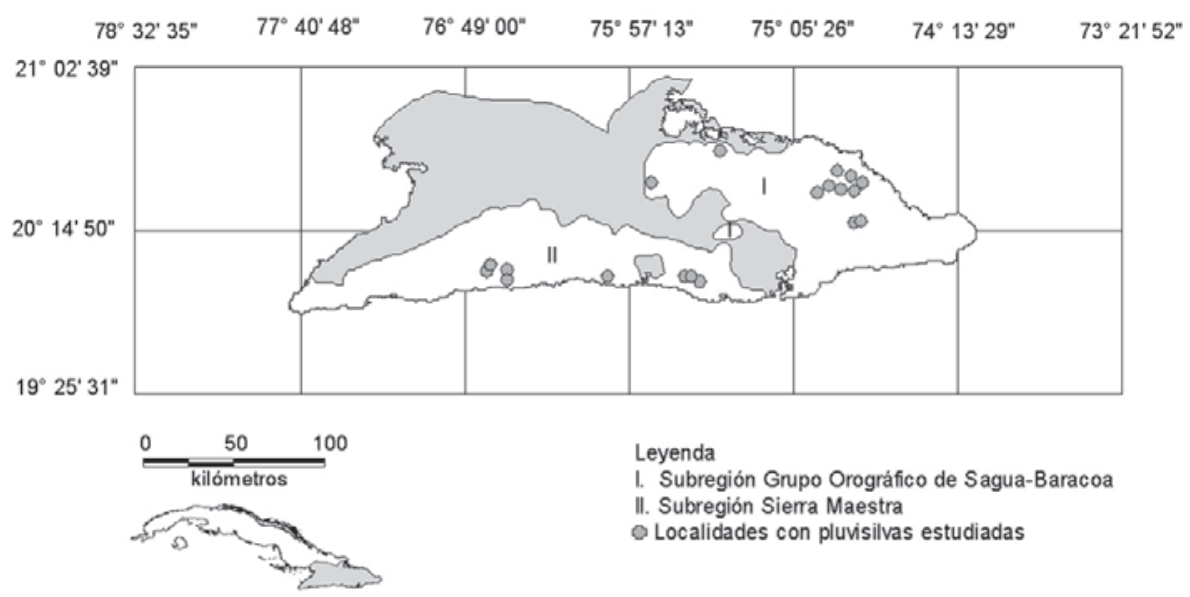

Figura 1. Región Oriental de Cuba, subregiones y localidades donde se estudiaron las pluvisilvas.

Figure 1. Oriental Region of Cuba, sub-regions and localities where rainforest were studied.

1998, Barreto 1998, González \& Bisse 1998, Panfet 1998, 2005, Rankin 1998, 2003, 2005, Dietrich 2000, Moya \& Leiva 2000, Gutiérrez 2000, 2002, Urquiola et al. 2000, Saralegui 2000, 2004, Dressler 2000, Rodríguez 2000, Sierra 2000, Thiv 2002, Greuter 2002, Beyra 2002, Mai 2003, 2005, Méndez 2003, González \& Sierra 2004, Albert 2005, Pérez 2005). Además, se tuvo en cuenta la base de datos de los endemismos cubanos que se encuentra en el Museo Nacional de Historia Natural, del Ministerio de Ciencia, Tecnología y Medio Ambiente en la Ciudad de La Habana.

Cada endemismo se clasificó según el criterio de López et al. (1994a), pero se adecuó al Sector Cuba Oriental que es el área de trabajo. Los tipos de endemismos son los siguientes:

-Endemismos totales o cubanos: el total de endemismos cubanos que está presente en el Sector Cuba Oriental.

-Endemismos sectoriales: aquella parte de los endemismos totales que sólo viven en el Sector Cuba Oriental.

-Endemismos estrictos o distritales: aquellos conocidos sólo de un distrito del Sector Cuba Oriental.

-Endemismos multidistritales: aquellos que viven en más de un distrito del Sector Cuba Oriental. -Endemismos multisectoriales: aquellos viven en más de un sector, o sea en Cuba Oriental y cualquier otro sector fitogeográfico del país.

Los endemismos se ubicaron en las diferentes unidades fitogeográficas de Cuba según Samek (1973), modificado por López et al. (1993), como se muestra en la Figura 1. En los casos donde los nombres de los distritos eran diferentes, se escribió entre paréntesis el más antiguo (Samek 1973).

Dicha ubicación en las unidades fitogeográficas se logró a partir de las localidades de colectas que se observaron en las fichas de los ejemplares consultados en los herbarios cubanos (HAC, HAJB y HACC) y los datos encontrados en la base de datos ya mencionada. De forma adicional se revisaron los artículos de Montes et al. (1985) y González-Torres et al. (2004) porque contienen especies en distritos de interés que también están presentes en las pluvisilvas.

La clasificación de las disyunciones (separación del área geográfica de un taxón dado en varias áreas independientes) se realizó según el criterio de Borhidi (1991). En este caso no se incluyó la distribución multisectorial, debido a que dicho autor concibió la separación de las áreas por causa ecológica y no geográfica. Se define como distribución trisectorial aquélla donde un taxón se distribuye en los tres sectores fitogeográficos, como distribución bisectorial, aquélla donde un taxón se encuentra sólo en dos sectores y como bipolaridad, aquélla donde 
Gayana Bot. 66(2), 2009

un taxón se encuentra en los dos sectores extremos (Cuba Occidental y Cuba Oriental).

ESPECIES NO ENDÉMICAS

Se hizo una separación entre los taxones que pertenecen a las pluvisilvas de la Subregión Grupo Orográfico Sagua-Baracoa y la Subregión Sierra Maestra a partir del criterio de Núñez \& Viña (1989) (Fig. 2), porque se asumió que dichas subregiones tienen un origen diferente, de forma tal que se consideró la edad geológica de éstas, a partir del criterio de Formell(1989). Como la separación físicogeográfica en esas dos subregiones coincide casi en su totalidad con la fitogeográfica (Samek 1973, modificado por López et al. 1993) se hará referencia a los subsectores Nor Oriental y Sur Oriental en este tipo de análisis. La distribución de cada taxón se obtuvo a partir de las obras que se utilizaron para la identificación de los taxones. La ubicación de cada taxón en los reinos y regiones del mundo se realizó a partir del criterio de Schmidt (1969). En la Región Caribe se utilizó el de Borhidi \& Muñiz (1986), ratificado por Borhidi (1991, 1996).

Centros de origen y evolución de las familias Los centros de origen y evolución de las familias se definieron a partir de Gentry (1982). No se tuvieron en cuenta para ese análisis a las familias Boraginaceae, Burseraceae, Lamiaceae, Linaceae, Sabiaceae, Turneraceae y Urticaceae porque sus centros de origen se definieron con dudas por Gentry (1982); por ello se introdujo el término "Origen dudoso" que aparece en la Tabla VIII. Tampoco se tuvieron en cuenta Cecropiaceae, Chrysobalanaceae, Cleomaceae, Hypericaceae y Viscaceae, debido a que fueron segregadas de otras familias cuyos centros de origen ya estaban definidos. Por otra parte, para Agavaceae y Smilacaceae se asumió el centro de origen de Liliaceae, por estar relacionadas.

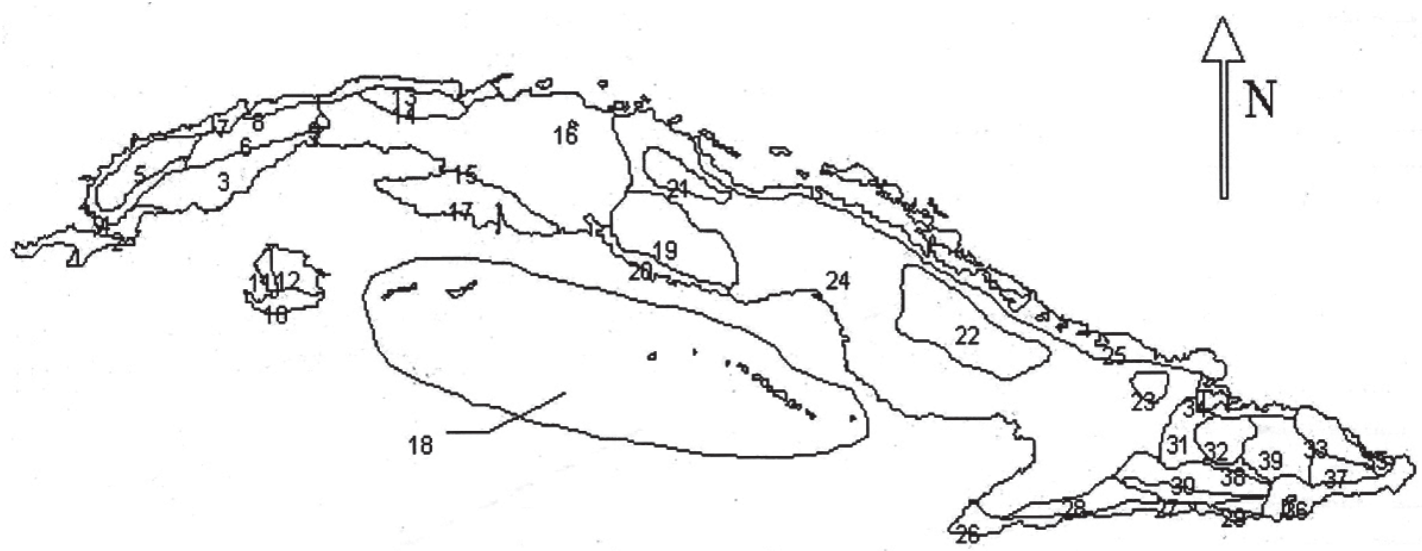

Figura 2. Distritos fitogeográficos de Cuba, según Samek (1973) modificado por López et al. (1993).

Figure 2. Phytogeographic districts of Cuba, according to Samek (1973) modified by López et al. (1993).

CUBA OCCIDENTAL: 1. Guanahacabibes (Península de Guanahacabibes), 2. Guane (Arenas blancas), 3. Pinar del Río (Centro Meridional de Pinar del Río), 4. Pizarras (Alturas de Pizarra), 5. Mogotes, 6. Sierra del Rosario, 7. Cajálbana (Sierra de Cajálbana), 8. Bahía Honda-Cabañas (Colinas de Bahía Honda-Cabañas), 9. Anafe (Sierra de Anafe), 10. Sur de Pinos (Meridional de Isla de Pinos), 11. Los Indios (Los Indios-Siguanea), 12. Centro de Pinos (Central de la Isla de Pinos). CUBA CENTRAL: 13. Habana-Matanzas (Costa Norte de Habana-Matanzas), 14. Habana-Limonar (Colinas de Habana-Limonar), 15. Llanura Centro-Occidental. 16. Motembo, 17. Zapata, 18. Cayería Sur, 19. Guamuhaya (Escambray), 20. Cienfuegos (Costero de Trinidad), 21. Santa Clara, 22. Camagüey (Serpentinitas de Camagüey). 23. Holguín (Serpentinitas de Holguín). 24. Llanura Centro Oriental (Llanuras y Colinas de Cuba Centro-Oriental). 25. Costa Centro-Oriental (Costa Norte Centro-Oriental). CUBA ORIENTAL: 26. Cabo Cruz-Baconao (Media Luna-Cabo Cruz-Baconao), 27. Promontorios de la Sierra Maestra, 28. Cordillera del Turquino, 29. Gran Piedra, 30. Valle Central (Valle Central de Oriente), 31. Sierra de Nipe, 32. Sierra de Cristal, 33. Baracoa (Serpentinitas de Moa-Toa-Baracoa), 34. Bahía de Nipe (Bahía de Nipe-Bahía de Cebollas), 35. Baracoa, 36. Maisí-Guantánamo (Costa Meridional de Maisí-Guantánamo), 37. Sierra de Imías, 38. Colinas de Oriente, 39. Santa Catalina. 
Fitogeografía de las pluvisilvas de Cuba Oriental: Martínez-Quesada, E.

\section{DendRogramas}

Para obtener los dendrogramas se confeccionaron tres matrices de presencia y ausencia de especies, una para todos los endemismos que se ubicaron en cada uno de los distritos fitogeográficos según Samek (1973), modificado por López et al. (1993) y las dos restantes para los no endemismos de la Región Caribe presentes en las pluvisilvas de los subsectores Nor Oriental y Sur Oriental, las que sirvieron de base para la aplicación del programa estadístico Statistica 98 (versión 6.0 para Windows).

CLASIFICACIÓN DE LAS PLUVISILVAS

La clasificación de las pluvisilvas se realizó de acuerdo con el criterio de Reyes \& Acosta (2005), quienes consideran cuatro tipos en el Parque Nacional Alejandro de Humboldt, las que pueden encontrarse en toda la Subregión Grupo Orográfico de Sagua-Baracoa (Pluvisilva de baja altitud sobre complejo metamórfico, Pluvisilva submontana sobre complejo metamórfico, Pluvisilva de baja altitud y submontana sobre ofiolitas y Pluvisilva submontana sobre suelos de mal drenaje); mientras que en la Subregión Sierra Maestra se utilizó la clasificación de Reyes (2006), el que reconoce sólo un tipo (Pluvisilva montana). En este caso el término pluvisilva es equivalente a Bosque pluvial, empleado internacionalmente.

Area de estudio

Para definir Región Oriental se tuvieron en cuenta los mapas que expusieron Núñez (1954), Cañas (1978), Mateo \& Acevedo (1989) y Núñez \& Viña (1989). Esta se define como la unidad natural-antrópica que se extiende por el extremo este de Cuba, que va desde el centro del Golfo de Guacanayabo, por el sur hasta la Bahía de Banes, por el norte. Limita al norte con la Región Central (en su mayor parte con las subregiones Llanura de Camagüey y Grupo Orográfico de Maniabón), al noreste con el Océano Atlántico, al este con el Paso de los Vientos, al sur con el Mar Caribe y al oeste con el mencionado Golfo de Guacanayabo. Esta región consta de 11 subregiones como parte del país emergido (Núñez \& Viña 1989), dos de las cuales (Grupo Orográfico de Sagua-Baracoa y Sierra Maestra) contienen las pluvisilvas (Fig. 1).

\section{RESULTADOS}

ANÁLISIS DE LA DISTRIBUCIÓN DE LAS ESPECIES ENDÉMICAS POR DISTRITOS FITOGEOGRÁFICOS Un total de 459 especies endémicas se distribuyeron en el Sector Cuba Oriental (sectoriales), mientras que sólo 69 se compartieron con otros sectores fitogeográficos (multisectoriales) y 42 se observaron en los tres sectores (totales). De las consideradas sectoriales, 274 se encontraron distribuidas en más de un distrito (multidistritales orientales), en tanto que 185 se limitaron a uno solo (distritales) (Tabla I).

En Cuba Oriental, Subsector Nor-Oriental, la mayoría de los endemismos se hallaron en los distritos Serpentinitas de Moa-Toa-Baracoa (418), Sierra de Nipe (192) y Sierra de Cristal (189), pero el primero de éstos acumuló más distritales (120). Por otra parte, en el sur-oriental el distrito montañoso de la Cordillera del Turquino fue el único que se destacó con 167 endemismos y 43 distritales (Tablas I y II). Los endemismos distritales se hallaron en 54 familias, sobre todo en Rubiaceae, Melastomataceae, Myrtaceae, Euphorbiaceae, Asteraceae y Orchidaceae. En el distrito Serpentinitas de Moa-Toa-Baracoa sobresalieron por el número de taxones infragenéricos de este tipo las familias Rubiaceae (15), Melastomataceae (12), Myrtaceae y Euphorbiaceae (diez cada una) y Asteraceae (siete de ellos). Por otra parte, en el distrito montañoso de la Cordillera del Turquino tuvieron importancia Euphorbiaceae y Orchidaceae, ambas con cinco y Melastomataceae y Rubiaceae con cuatro.

El Subsector Cuba Centro-Oriental presentó los mayores porcentajes de especies compartidas con Cuba Oriental y en éste el Distrito Escambray fue el más importante. Por otra parte, hubo una menor cantidad de especies compartidas con Cuba Occidental que se acumularon más en el Subsector Pinar del Río, sobre todo en el Distrito Sierra del Rosario y luego en Cajálbana (Tablas III y IV).

Las relaciones de afinidad florística entre los distritos de Cuba Oriental y los de otros sectores también se pudieron observar a partir del agrupamiento de estas unidades fitogeográficas en el dendrograma de la Figura 3. En Cuba Oriental se halló una relación muy estrecha entre la Sierra de Nipe y la Sierra de Cristal y junto a éstos, aunque de forma separada, se apreció el distrito 
Gayana Bot. 66(2), 2009

Serpentinitas de Moa-Toa-Baracoa. Otros distritos de importancia por la acumulación de especies endémicas compartidas se apreciaron de forma aislada, como Santa Catalina y el montañoso de la Cordillera del Turquino. Además, hubo cierta relación entre este último y el de Gran Piedra. Por otra parte, en el sector Cuba Central, el distrito Escambray tuvo más relación con los distritos de Cuba Oriental y a su vez todos ellos con Sierra del Rosario, en Cuba Occidental.

TABLA I. Endemismos distritales presentes en las pluvisilvas de la Región Oriental de Cuba (Sector Cuba Oriental).

TABLE I. Distrital endemism on rainforest of Eastern Cuba (Cuba Oriental).

Distritos: 26. Media Luna-Cabo Cruz-Baconao, 27. Promontorios de la Sierra Maestra, 28. Cordillera del Turquino, 29. Gran Piedra, 31. Sierra de Nipe, 32. Sierra de Cristal, 33. Serpentinitas de Moa-Toa-Baracoa, 37. Sierra de Imías, 39 Santa Catalina.

\begin{tabular}{|c|c|c|c|c|c|c|c|c|c|}
\hline \multirow[t]{2}{*}{ Taxones infragenéricos } & \multicolumn{9}{|c|}{ Distritos fitogeográficos } \\
\hline & 39 & 37 & 33 & 32 & 31 & 29 & 28 & 27 & 26 \\
\hline Acrosynanthus latifolius Standl. & & & $\mathrm{x}$ & & & & & & \\
\hline Acrosynanthus revolutus Urb. & & & $\mathrm{x}$ & & & & & & \\
\hline Acrosynanthus trachyphyllus Standl. & & & $\mathrm{x}$ & & & & & & \\
\hline Angadenia moaensis Lippold & & & $\mathrm{x}$ & & & & & & \\
\hline Annona cristalensis (Alain) Bisse & & & & $\mathrm{x}$ & & & & & \\
\hline Antirhea orbicularis Alain & & & $\mathrm{x}$ & & & & & & \\
\hline Antirhea pedicellaris Borhidi \& Bisse & & & $\mathrm{x}$ & & & & & & \\
\hline Arthrostylidium pinifolium Catasús & & & $\mathrm{x}$ & & & & & & \\
\hline Begonia maestrensis Urb. & & & & & & & $\mathrm{x}$ & & \\
\hline Bonnetia cubensis (Britton) R.A.Howard & & & $\mathrm{x}$ & & & & & & \\
\hline Buxus aneura Urb. & & & & & $\mathrm{x}$ & & & & \\
\hline Buxus crassifolia (Britton) Urb. var. crassifolia & & & $\mathrm{x}$ & & & & & & \\
\hline Buxus foliosa (Britton) Urb. & & & $\mathrm{x}$ & & & & & & \\
\hline Buxus vaccinioides (Britton) Urb. & & & $\mathrm{x}$ & & & & & & \\
\hline Calycogonium ellipticum C. Wright \& Sauvalle & & & $\mathrm{x}$ & & & & & & \\
\hline Calycogonium heterophyllum Naudin ssp. heterophyllum & & & $\mathrm{x}$ & & & & & & \\
\hline $\begin{array}{l}\text { Calycogonium moanum (Borhidi \& O.Muñiz) Borhidi \& } \\
\text { O.Muñiz }\end{array}$ & & & $\mathrm{x}$ & & & & & & \\
\hline $\begin{array}{l}\text { Calycogonium rosmarinifolium Griseb. ssp. } \\
\text { brachyphyllum Borhidi \& O.Muñiz }\end{array}$ & & & & $\mathrm{x}$ & & & & & \\
\hline Calycolpus cristalense (Urb.) Bisse & & & & $\mathrm{x}$ & & & & & \\
\hline Calyptranthes apoda Urb. & & & $\mathrm{x}$ & & & & & & \\
\hline Calyptranthes compressa Urb. & & & $\mathrm{x}$ & & & & & & \\
\hline Calyptranthes linearis Alain & & & $\mathrm{x}$ & & & & & & \\
\hline Calyptranthes mirabilis Bisse \& A.Rodr. & & & $\mathrm{x}$ & & & & & & \\
\hline Calliandra enervis (Britton) Urb. & & & $\mathrm{x}$ & & & & & & \\
\hline Callicarpa leonis Moldenke & & & $\mathrm{x}$ & & & & & & \\
\hline $\begin{array}{l}\text { Casasia nigrescens (Griseb.) C.Wright ex Urb. ssp. } \\
\text { moaënsis Borhidi \& O.Muñiz }\end{array}$ & & & $\mathrm{x}$ & & & & & & \\
\hline Cestrum pinetorum Britton & & & $\mathrm{x}$ & & & & & & \\
\hline Cissus grisebachii Planch. & & & $\mathrm{x}$ & & & & & & \\
\hline Cissus nipensis Urb. & & & & & $\mathrm{x}$ & & & & \\
\hline Cissus subavenia Planch. & & & $\mathrm{x}$ & & & & & & \\
\hline Clusia alainii Borhidi & & & $\mathrm{x}$ & & & & & & \\
\hline Clusia callosa Britton \& P.Wilson & & & $\mathrm{x}$ & & & & & & \\
\hline Clusia moaensis Borhidi \& O.Muñiz & & & $\mathrm{x}$ & & & & & & \\
\hline
\end{tabular}


Fitogeografía de las pluvisilvas de Cuba Oriental: Martínez-Quesada, E.

\begin{tabular}{|c|c|c|c|c|c|c|c|c|c|}
\hline \multirow[t]{2}{*}{ Taxones infragenéricos } & \multicolumn{9}{|c|}{ Distritos fitogeográficos } \\
\hline & 39 & 37 & 33 & 32 & 31 & 29 & 28 & 27 & 26 \\
\hline Clusia munizii Borhidi & & & $\mathrm{x}$ & & & & & & \\
\hline Coccoloba baracoensis Schmidt. & & & $\mathrm{x}$ & & & & & & \\
\hline Coccoloba oligantha Alain & & & $\mathrm{x}$ & & & & & & \\
\hline Coccoloba toaensis Alain & & & $\mathrm{x}$ & & & & & & \\
\hline Cubacroton maestrensis Alain & & & & & & & $\mathrm{x}$ & & \\
\hline Cyrilla cubensis P.Wilson & & & $\mathrm{x}$ & & & & & & \\
\hline Dendrophthora excisa Urban & & & & & & & $\mathrm{x}$ & & \\
\hline Dilomilis bissei H.A.Dietr. & & & $\mathrm{x}$ & & & & & & \\
\hline Dinema cubincola (Borhidi) A.Dietr. & & & $\mathrm{x}$ & & & & & & \\
\hline Ditta maestrense Borhidi & & & & & & & $\mathrm{x}$ & & \\
\hline Dorstenia howardii León & & & $\mathrm{x}$ & & & & & & \\
\hline Dracaena cubensis Victorin & & & $\mathrm{x}$ & & & & & & \\
\hline Drosera moaensis C.Panfet & & & $\mathrm{x}$ & & & & & & \\
\hline Duranta wrightii Moldenke & & & $\mathrm{x}$ & & & & & & \\
\hline Elaeodendron lippoldii Bisse & & & & & & & $\mathrm{x}$ & & \\
\hline Eugenia alainii Borhidi & & & $\mathrm{x}$ & & & & & & \\
\hline Eugenia oligadenia Urb. & & & & & & & $\mathrm{x}$ & & \\
\hline $\begin{array}{l}\text { Euphorbia helenae Urb ssp. grandifolia Borhidi \& } \\
\text { O.Muñiz }\end{array}$ & & & $\mathrm{x}$ & & & & & & \\
\hline Euphorbia helenae ssp. helenae & & & $\mathrm{x}$ & & & & & & \\
\hline Euphorbia munizii Borhidi & & & $\mathrm{x}$ & & & & & & \\
\hline Exostema dumosum Alain & & & $\mathrm{x}$ & & & & & & \\
\hline Exostema obovatum Alain & & & $\mathrm{x}$ & & & & & & \\
\hline Galactia earlei ssp. toaensis Borhidi \& O.Muñiz & & & $\mathrm{x}$ & & & & & & \\
\hline Gochnatia shaferi (Britton) Jervis \& Alain & & & & & $\mathrm{x}$ & & & & \\
\hline Guapira rufescens Lundell var. rufescens & & & $\mathrm{x}$ & & & & & & \\
\hline Gundlachia cubana Britton \& S.F.Blake & & & $\mathrm{x}$ & & & & & & \\
\hline Harpalyce baracoensis Borhidi \& O.Muñiz & & & $\mathrm{x}$ & & & & & & \\
\hline Harpalyce ekmanii Urb. & & & $\mathrm{x}$ & & & & & & \\
\hline Helietta cubensis Monachino \& Moldenke & & & $\mathrm{x}$ & & & & & & \\
\hline Henriettea acunae (Alain) Alain & & & $\mathrm{x}$ & & & & & & \\
\hline Henriettea ekmanii (Urb.) Alain & & & & & & & $\mathrm{x}$ & & \\
\hline Henriettea gibberosa (Urb.) Alain & & & & & & & $\mathrm{x}$ & & \\
\hline Henriettea squamata (Alain) Alain & & & $\mathrm{x}$ & & & & & & \\
\hline Heptanthus shaferi Britton & & & $\mathrm{x}$ & & & & & & \\
\hline Hottea moana (Borhidi \& O.Muñiz) Borhidi & & & $\mathrm{x}$ & & & & & & \\
\hline Ilex valenzuelana Alain & & & & & & & $\mathrm{x}$ & & \\
\hline Jacquinia acunana Borhidi \& O.Muñiz & & & $\mathrm{x}$ & & & & & & \\
\hline Jacquinia sessiliflora Alain & & & & $\mathrm{x}$ & & & & & \\
\hline $\begin{array}{l}\text { Koanophyllon ayapanoides (Griseb.) R.M.King \& } \\
\text { H.Rob. }\end{array}$ & & & $\mathrm{x}$ & & & & & & \\
\hline Krokia albescens (Alain) Bisse & & & $\mathrm{x}$ & & & & & & \\
\hline Lagetta valenzuelana A.Rich. ssp. pauciflora (Urb.) Noa & & & & & $\mathrm{x}$ & & & & \\
\hline Laplacea angustifolia (Britton \& P.Wilson) O.C.Schmidt & & & & & & & $\mathrm{x}$ & & \\
\hline Lepanthes acunae Hespenh. & & & & & & & & $\mathrm{x}$ & \\
\hline Lepanthes blepharantha Schltr. & & & & & & & & $\mathrm{x}$ & \\
\hline Lepanthes ekmanii Schtr. & & & & & & & & $\mathrm{x}$ & \\
\hline Lepanthes fractiflexa Ames \& C.Schweinf. & & & & & & & $\mathrm{x}$ & & \\
\hline Lepanthes pergracilis Schltr. & & & & & & & $\mathrm{x}$ & & \\
\hline Leucocroton linearifolius Britton & & & $\mathrm{x}$ & & & & & & \\
\hline Leucocroton pachyphyllus Urb. & & & $\mathrm{x}$ & & & & & & \\
\hline
\end{tabular}


Gayana Bot. 66(2), 2009

\begin{tabular}{|c|c|c|c|c|c|c|c|c|c|}
\hline \multirow[t]{2}{*}{ Taxones infragenéricos } & \multicolumn{9}{|c|}{ Distritos fitogeográficos } \\
\hline & 39 & 37 & 33 & 32 & 31 & 29 & 28 & 27 & 26 \\
\hline Linum cubense Bisse & & & $\mathrm{x}$ & & & & & & \\
\hline Lobelia oxyphylla Urb. & & & & & & & $\mathrm{x}$ & & \\
\hline Machaerina filifolia Griseb. & & & $\mathrm{x}$ & & & & & & \\
\hline Magnolia cacuminicola Bisse & & $\mathrm{x}$ & & & & & & & \\
\hline Malpighia martiana Acuña \& Roig & & & $\mathrm{x}$ & & & & & & \\
\hline $\begin{array}{l}\text { Maytenus buxifolia (A.Rich.) Griseb. ssp. monticola } \\
\text { Borhidi \& O.Muñiz }\end{array}$ & & & & & & & $\mathrm{x}$ & & \\
\hline Maytenus maestrensis Urb. & & & & & & & $\mathrm{x}$ & & \\
\hline Mecranium obtusifolium Cogn. & & & $\mathrm{x}$ & & & & & & \\
\hline Meliosma oppositifolia Griseb. & & & $\mathrm{x}$ & & & & & & \\
\hline Miconia bucherae Alain & & & $\mathrm{x}$ & & & & & & \\
\hline Miconia pteroclada Urb. & & & & & & & $\mathrm{x}$ & & \\
\hline Moacroton tetramerus Borhidi \& O.Muñiz & & & $\mathrm{x}$ & & & & & & \\
\hline Morinda moaensis Alain & & & $\mathrm{x}$ & & & & & & \\
\hline Mosiera calycolpoides (Griseb.) Borhidi & & & $\mathrm{x}$ & & & & & & \\
\hline Mozartia gundlachii (Krug \& Urb.) Urb. & & & $\mathrm{x}$ & & & & & & \\
\hline Myrsine bissei Panfet & & & $\mathrm{x}$ & & & & & & \\
\hline Myrsine cristalensis Borhidi & & & & $\mathrm{x}$ & & & & & \\
\hline Myrica cacuminis Britton \& P.Wilson & & & & & & & $\mathrm{x}$ & & \\
\hline Nectandra reticularis Britton \& P.Wilson & & & & & & & $\mathrm{x}$ & & \\
\hline Neobracea ekmanii Urb. & & & $\mathrm{x}$ & & & & & & \\
\hline Ocotea cristalensis Bisse & & & & $\mathrm{x}$ & & & & & \\
\hline Ossaea baracoënsis Borhidi \& O.Muñiz var. baracoënsis & & & $\mathrm{x}$ & & & & & & \\
\hline Ossaea elliptica Alain & & & $\mathrm{x}$ & & & & & & \\
\hline Ossaea granulata Urb. & & & $\mathrm{x}$ & & & & & & \\
\hline Ossaea moaensis Alain & & & $\mathrm{x}$ & & & & & & \\
\hline $\begin{array}{l}\text { Ossaea nipensis Britton \& P.Wilson var. longipetiolata } \\
\text { Borhidi \& O.Muñiz }\end{array}$ & & & & & $\mathrm{x}$ & & & & \\
\hline Ossaea nipensis var. nipensis & & & & & $\mathrm{x}$ & & & & \\
\hline Ossaea pulchra Alain & & & $\mathrm{x}$ & & & & & & \\
\hline Ossaea pulverulenta Urb. & & & & & & & $\mathrm{x}$ & & \\
\hline Paepalanthus riparius Moldenke & & & $\mathrm{x}$ & & & & & & \\
\hline Passiflora maestrensis Duharte & & & & & & & $\mathrm{x}$ & & \\
\hline Pentacalia pachypoda (Greenm.) Borhidi & & & $\mathrm{x}$ & & & & & & \\
\hline Peperomia cuspidata Dahlst. & & & & & & & & & $\mathrm{x}$ \\
\hline Pera longipes Britton \& P.Wilson & & & $\mathrm{x}$ & & & & & & \\
\hline Phyllanthus incrustatus Urb. & & & $\mathrm{x}$ & & & & & & \\
\hline Pimenta oligantha (Urb.) Burret & & & $\mathrm{x}$ & & & & & & \\
\hline Pinguicula lignicola Barnhart & & & $\mathrm{x}$ & & & & & & \\
\hline Piper obtusum C.DC. & & & & & & & $\mathrm{x}$ & & \\
\hline Pisonia byrsonimifolia Heimerl \& Ekman & & & $\mathrm{x}$ & & & & & & \\
\hline Pleurathalis denticulata Cogn. & & & & & & & & & $\mathrm{x}$ \\
\hline Pleurothallis obliquipetala Acuña \& Schweinf. & & & & & & & $\mathrm{x}$ & & \\
\hline Pleurothallis odontotepala Rchb.f. & & & & & & & $\mathrm{x}$ & & \\
\hline Plinia baracoensis Borhidi & & & $\mathrm{x}$ & & & & & & \\
\hline Pouteria cubensis Baehni & & & & & & & $\mathrm{x}$ & & \\
\hline Pouteria moaensis Alain & & & $\mathrm{x}$ & & & & & & \\
\hline Protium baracoense Bisse & & & $\mathrm{x}$ & & & & & & \\
\hline Protium fragans (Rose) Urb. & & & $\mathrm{x}$ & & & & & & \\
\hline Protium maestrense Bisse & & & & & & & $\mathrm{x}$ & & \\
\hline Psychotria ekmanii Urb. & & & & & & & $\mathrm{x}$ & & \\
\hline
\end{tabular}


Fitogeografía de las pluvisilvas de Cuba Oriental: Martínez-Quesada, E.

\begin{tabular}{|c|c|c|c|c|c|c|c|c|c|}
\hline \multirow[t]{2}{*}{ Taxones infragenéricos } & \multicolumn{9}{|c|}{ Distritos fitogeográficos } \\
\hline & 39 & 37 & 33 & 32 & 31 & 29 & 28 & 27 & 26 \\
\hline Psychotria moensis Britton \& P.Wilson & & & $\mathrm{x}$ & & & & & & \\
\hline Psychotria sphaeroidea Urb. & & & & & & & $\mathrm{x}$ & & \\
\hline Purdiaea moaensis Victorin & & & $\mathrm{x}$ & & & & & & \\
\hline Purdiaea ophiticola Victorin var. parvifolia Victorin & & & $\mathrm{x}$ & & & & & & \\
\hline Purdiaea shaferi Britton \& P.Wilson & & & $\mathrm{x}$ & & & & & & \\
\hline Purdiaea stenopetala Griseb. var. stenopetala & & & $\mathrm{x}$ & & & & & & \\
\hline $\begin{array}{l}\text { Ravenia shaferi P.Wilson var. ekmanii (Urb.) Borhidi \& } \\
\text { O.Muñiz }\end{array}$ & & & $\mathrm{x}$ & & & & & & \\
\hline $\begin{array}{l}\text { Reynosia mucronata Griseb. ssp. nipensis Borhidi \& } \\
\text { O.Muñiz }\end{array}$ & & & & & $\mathrm{x}$ & & & & \\
\hline Rondeletia bracteosa Borhidi \& M.Fernández & & & $\mathrm{x}$ & & & & & & \\
\hline Rondeletia intermixta Britton ssp. intermixta & & & & & & $\mathrm{x}$ & & & \\
\hline $\begin{array}{l}\text { Rondeletia intermixta ssp. turquinensis M.Z.Fernández } \\
\& \text { Borhidi }\end{array}$ & & & & & & & $\mathrm{x}$ & & \\
\hline Rondeletia vazquezii Borhidi \& O.Muñiz & $\mathrm{x}$ & & & & & & & & \\
\hline Rubus turquinensis Rydb. & & & & & & & $\mathrm{x}$ & & \\
\hline Sapium erythrospermum (Griseb.) Muell.Arg. & & & & & & & $\mathrm{x}$ & & \\
\hline Sapium maestrense Urb. & & & & & & & $\mathrm{x}$ & & \\
\hline Sapium moaense Alain & & & $\mathrm{x}$ & & & & & & \\
\hline Sapium parvifolium Alain & & & $\mathrm{x}$ & & & & & & \\
\hline Schmidtottia monantha Urb. & & & $\mathrm{x}$ & & & & & & \\
\hline Schmidtottia multiflora Urb. & & & $\mathrm{x}$ & & & & & & \\
\hline Schmidtottia sessilifolia (Britton) Urb. & & & $\mathrm{x}$ & & & & & & \\
\hline Schmidtottia shaferi (Standl.) Urb. var. micarensis Alain & & & & $\mathrm{x}$ & & & & & \\
\hline Schoepfia cubensis Britton \& P.Wilson & & & $\mathrm{x}$ & & & & & & \\
\hline Schoepfia stenophylla Urb. & & & & & & & $\mathrm{x}$ & & \\
\hline Scolosanthus wrightianus (Griseb.) S.H.Wright ex Sauv. & & & $\mathrm{x}$ & & & & & & \\
\hline Senecio pachylepis Greenm. & & & $\mathrm{x}$ & & & & & & \\
\hline $\begin{array}{l}\text { Senna gundlachii (Urb.) H.S.Irwin \& Barneby ssp. } \\
\text { esmeraldensis (Alain) Barreto \& Yakov. }\end{array}$ & & & $\mathrm{x}$ & & & & & & \\
\hline $\begin{array}{l}\text { Sideroxylon ekmanianum (Urb.) Bisse, J.E.Gut. \& } \\
\text { Iglesias }\end{array}$ & & & & & & & $\mathrm{x}$ & & \\
\hline Siphocampylus patens Griseb. & & & $\mathrm{x}$ & & & & & & \\
\hline Solonia reflexa Urb. & & & & & & & $\mathrm{x}$ & & \\
\hline Spathelia vernicosa Planchon & & & $\mathrm{x}$ & & & & & & \\
\hline Spathelia wrightii Victorin & & & $\mathrm{x}$ & & & & & & \\
\hline Spermacoce oligantha Urb. & & & & & & & $\mathrm{x}$ & & \\
\hline Symplocos lindeniana Krug \& Urb. & & & & & & & $\mathrm{x}$ & & \\
\hline Tabebuia clementis Alain & & & $\mathrm{x}$ & & & & & & \\
\hline $\begin{array}{l}\text { Talauma minor Urb. ssp. orbiculata (Britton \& P.Wilson) } \\
\text { Borhidi }\end{array}$ & & & & & & & $\mathrm{x}$ & & \\
\hline Tapura orientensis Köehler & & & $\mathrm{x}$ & & & & & & \\
\hline Terminalia orientensis Monach. & & & $\mathrm{x}$ & & & & & & \\
\hline Ternstroemia microcalyx Krug \& Urb. & & & & & & & $\mathrm{x}$ & & \\
\hline Ternstroemia moensis Borhidi & & & $\mathrm{x}$ & & & & & & \\
\hline Tolumnia acunae (M.A.Díaz) Nir. & & & & & & & $\mathrm{x}$ & & \\
\hline Vaccinium leonis Acuña \& Roig & & & & & & & $\mathrm{x}$ & & \\
\hline Vaccinium shaferi Acuña \& Roig. & & & $\mathrm{x}$ & & & & & & \\
\hline Varronia duartei (Borhidi \& O.Muñiz) Borhidi & & & $\mathrm{x}$ & & & & & & \\
\hline Varronia iberica (Urb.) Borhidi & & & $\mathrm{x}$ & & & & & & \\
\hline Varronia longipedunculata Britton \& P.Wilson & & & & & & & $\mathrm{x}$ & & \\
\hline
\end{tabular}


Gayana Bot. 66(2), 2009

\begin{tabular}{|c|c|c|c|c|c|c|c|c|c|}
\hline \multirow[t]{2}{*}{ Taxones infragenéricos } & \multicolumn{9}{|c|}{ Distritos fitogeográficos } \\
\hline & 39 & 37 & 33 & 32 & 31 & 29 & 28 & 27 & 26 \\
\hline Vernonia maestralis Ekman ex Urb. & & & & & & & $\mathrm{x}$ & & \\
\hline Vernonia segregata Gleason & & & $\mathrm{x}$ & & & & & & \\
\hline $\begin{array}{l}\text { Wallenia jacquinioides (Griseb.) Mez ssp. montecristensis } \\
\text { Panfet \& Ventosa }\end{array}$ & & & $\mathrm{x}$ & & & & & & \\
\hline Wallenia lepperi Panfet \& Ventosa & & & $\mathrm{x}$ & & & & & & \\
\hline Wallenia maestrensis Panfet \& Ventosa & & & & & & & $\mathrm{x}$ & & \\
\hline Xylosma iberiensis J.E.Gut. & & & $\mathrm{x}$ & & & & & & \\
\hline Zanthoxylum lomincola (Urb.) Alain & & & $\mathrm{x}$ & & & & & & \\
\hline Zanthoxylum shaferi P.Wilson & & & $\mathrm{x}$ & & & & & & \\
\hline Zonanthus cubensis Griseb. & & & $\mathrm{x}$ & & & & & & \\
\hline
\end{tabular}

TABLA II. Distribución de los endemismos de las pluvisilvas estudiadas en Cuba Oriental.

TABLE II. Distribution of endemism from rainforest studied on Eastern Cuba.

\begin{tabular}{|c|c|c|}
\hline Unidad fitogeográfica & $\mathrm{N}^{\circ}$ taxones infragenéricos & Porcentaje \\
\hline \multicolumn{3}{|l|}{ SubSector Sur Oriental } \\
\hline Media Luna, Cabo Cruz, Baconao & 43 & 8 \\
\hline Promontorios de la Sierra Maestra & 38 & 7 \\
\hline Cordillera del Turquino & 167 & 29 \\
\hline Gran Piedra & 70 & 12 \\
\hline Subsector Valle Central de Oriente & 26 & 5 \\
\hline \multicolumn{3}{|l|}{ SubSECtOR Nor ORIENTAL } \\
\hline Sierra de Nipe & 192 & 34 \\
\hline Sierra de Cristal & 189 & 33 \\
\hline Serpentinitas de Moa- Toa-Baracoa & 418 & 73 \\
\hline Bahía de Nipe-Bahía de Cebollas & 37 & 6 \\
\hline Baracoa & 44 & 8 \\
\hline Maisí-Guantánamo & 51 & 9 \\
\hline Sierra de Imías & 36 & 6 \\
\hline Colinas de Oriente & 8 & 1 \\
\hline Santa Catalina & 140 & 25 \\
\hline
\end{tabular}


Fitogeografía de las pluvisilvas de Cuba Oriental: Martínez-Quesada, E.

TABLA III. Distribución de los endemismos de las pluvisilvas estudiadas en Cuba Central

TABLE III. Distribution of endemism from rainforest studied on Central Cuba

\begin{tabular}{|c|c|c|}
\hline Unidad fitogeográfica & $\begin{array}{c}\mathrm{N}^{\circ} \text { taxones } \\
\text { infragenéricos }\end{array}$ & Porcentaje \\
\hline \multicolumn{3}{|l|}{ Subsector Cuba Centro-Occidental } \\
\hline Costa Norte de Habana-Matanzas & 17 & 3 \\
\hline Colinas de Habana-Limonar & 32 & 7 \\
\hline Llanura Centro-Occidental & 27 & 5 \\
\hline Motembo & 16 & 3 \\
\hline Zapata & 14 & 2 \\
\hline Cayería Sur & 0 & 0 \\
\hline \multicolumn{3}{|l|}{ Subsector Cuba-Centro Oriental } \\
\hline Escambray & 44 & 8 \\
\hline Costero de Trinidad & 15 & 3 \\
\hline Serpentinitas de Santa Clara & 22 & 4 \\
\hline Serpentinitas de Camagüey & 23 & 4 \\
\hline Serpentinitas de Holguín & 22 & 4 \\
\hline Llanuras y colinas de Cuba Centro-Oriental & 24 & 4 \\
\hline Costa Norte Centro-Oriental & 25 & 4 \\
\hline
\end{tabular}

TABLA IV. Distribución de los endemismos de las pluvisilvas estudiadas en Cuba Occidental.

TABLE IV. Distribution of endemism from rainforest studied on Western Cuba.

\begin{tabular}{lcc}
\hline \multicolumn{1}{c}{ Unidad fitogeográfica } & $N^{\text {o }}$ taxones infragenéricos & Porcentaje \\
\hline Subsector PINAR DEL Río & 6 & 1 \\
Península de Guanahacabibes & 11 & 2 \\
Arenas blancas & 16 & 3 \\
Centro Meridional de Pinar del Río & 8 & 1 \\
Alturas de Pizarras & 17 & 3 \\
Los Mogotes & 43 & 8 \\
Sierra del Rosario & 28 & 5 \\
Cajálbana & 8 & 1 \\
Colinas de Bahía Honda- Cabañas & 8 & 1 \\
Anafe & & \\
SubSECTOR IsLA DE PINOS & 12 & 2 \\
Isla de Pinos & 3 & 1 \\
Los Indios & 7 & 1 \\
Centro de la Isla de Pinos & & \\
\hline
\end{tabular}




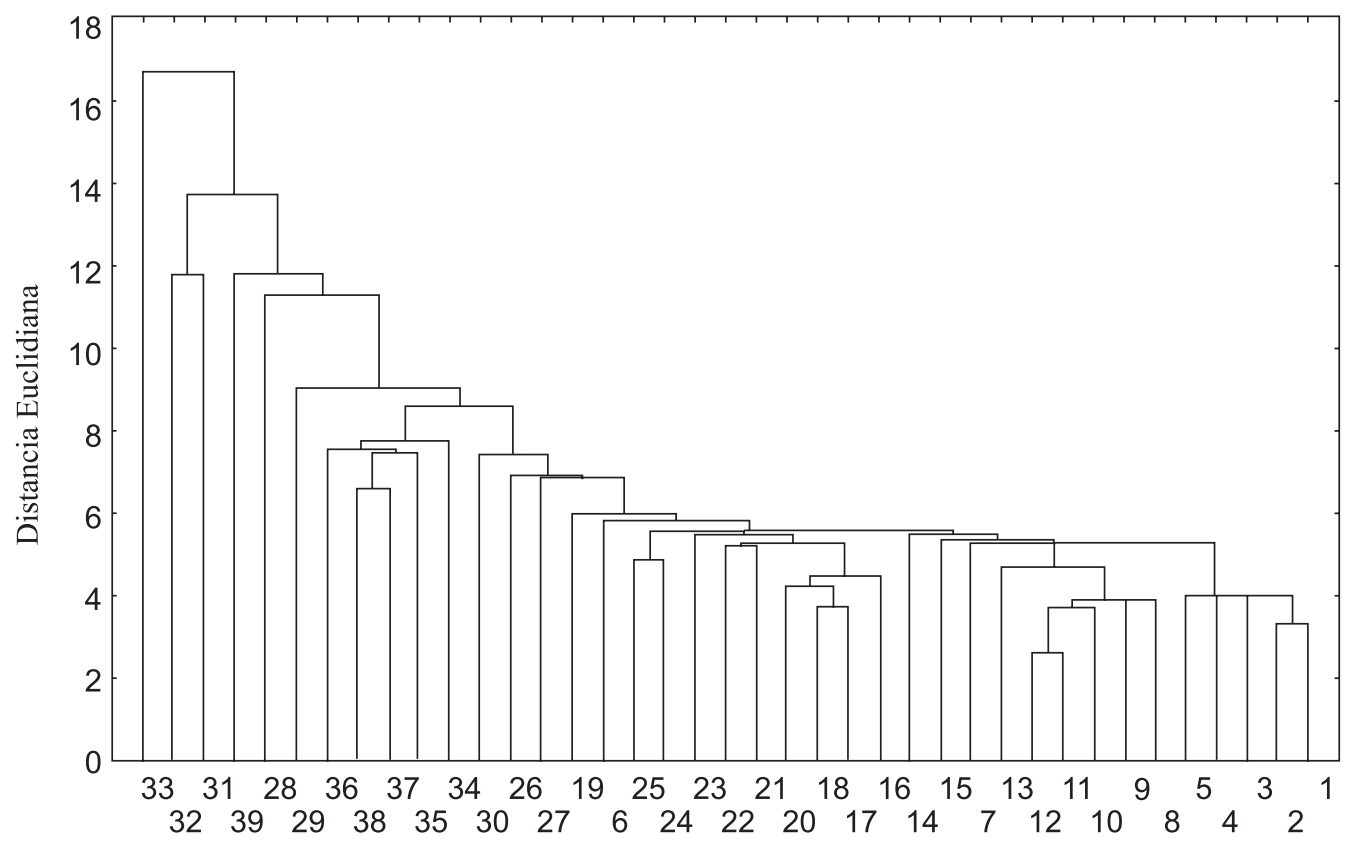

FIgURA 3. Dendrograma que muestra las relaciones entre todos los distritos fitogeográficos de Cuba, a partir de las espermatofitas presentes en las pluvisilvas de Cuba Oriental (Los números de los distritos corresponden con los de Samek 1973, modificados por López et al. 1993).

FIGURE 3. Dendrogram showing the relationships among all phytogeographic districts of Cuba, from spermatophytes on rainforest of Eastern Cuba. (Numbers of districts are those of Samek 1973, modified by López et al. 1993).

Distritos: 1. Península de Guanahacabibes 2. Arenas blancas, 3. Centro Meridional de Pinar del Río, 4. Alturas de Pizarras, 5. Mogotes, 6. Sierra del Rosario, 7. Cajálbana, 8. Colinas de Bahía Honda-Cabañas, 9. Sierra de Anafe, 10. Meridional de Isla de Pinos, 11. Los Indios-Siguanea, 12. Central de Isla de Pinos, 13. Costa Norte de HabanaMatanzas, 14. Colinas de Habana-Limonar, 15. Llanura Centro-Occidental, 16. Motembo, 17. Zapata, 18. Cayería Sur, 19. Escambray 20. Costero de Trinidad. 21. Santa Clara, 22. Serpentinitas de Camagüey, 23. Serpentinitas de Holguín, 24. Llanuras y colinas de Cuba Centro Oriental, 25. Costa Norte Centro-Oriental, 26. Media Luna-Cabo Cruz- Baconao, 27. Promontorios de la Sierra Maestra, 28. Cordillera del Turquino, 29. Gran Piedra, 30. Valle Central de Oriente, 31. Sierra de Nipe, 32. Sierra de Cristal, 33. Serpentinitas de Moa-Toa-Baracoa, 34. Bahía de Nipe-Bahía de Cebollas, 35. Baracoa, 36. Costa Meridional de Maisí-Guantánamo, 37. Sierra de Imías, 38. Colinas del borde septentrional del Valle Central de Oriente, 39 Santa Catalina.

\section{ESPECIES ENDÉMICAS DISYUNTAS}

Entre las especies endémicas se detectaron 39 que presentaron tres tipos de disyunción. Estas fueron la bipolaridad, la distribución bisectorial y la distribución trisectorial. Las especies con distribución bipolar constituyeron el 20\% de todas las disyuntas. En la distribución de éstas se observó que estuvieron en uno o dos distritos de Cuba Occidental y varios de Cuba Oriental. Las que se distribuyen en dos sectores (bisectoriales) resultaron las más numerosas y representaron el $41 \%$. La mayoría de ellas se observaron compartidas entre varios distritos del Subsector Nor-Oriental y los subsectores de Cuba Central. Aquellas con distribución en los tres sectores (trisectoriales) representaron el 38\%. Al igual que el caso anterior, la mayor parte de ellas se distribuyeron entre varios distritos del Subsector Nor-Oriental, los de Cuba Central y los de Cuba Occidental involucrados (Tabla V). 
Fitogeografía de las pluvisilvas de Cuba Oriental: Martínez-Quesada, E.

TABLA V. Taxones infragenéricos endémicos disyuntos.

TABLE V. Infrageneric endemic disjunct taxa.

Distritos: 1. Península de Guanahacabibes 2. Arenas blancas, 4. Alturas de Pizarras, 5. Mogotes, 6. Sierra del Rosario, 7. Cajálbana, 8. Colinas de Bahía Honda-Cabañas, 9. Sierra de Anafe, 10. Meridional de Isla de Pinos, 12. Central de Isla de Pinos, 13. Costa Norte de Habana-Matanzas, 14. Colinas de Habana-Limonar, 15. Llanura Centro-Occidental, 16. Motembo, 17. Zapata, 19. Escambray, 20. Costero de Trinidad, 21. Santa Clara, 22. Serpentinitas de Camaguey, 23. Serpentinitas de Holguín, 24. Llanuras y colinas de Cuba Centro Oriental, 25. Costa Norte Centro-Oriental, 26. Media Luna-Cabo Cruz-Baconao, 27. Promontorios de la Sierra Maestra, 28. Cordillera del Turquino, 29. Gran Piedra, 30. Valle Central de Oriente, 31. Sierra de Nipe, 32. Sierra de Cristal, 33. Serpentinitas de Moa-Toa-Baracoa, 34. Bahía de Nipe-Bahía de Cebollas, 35. Baracoa, 36. Costa Meridional de Maisí-Guantánamo, 37. Sierra de Imías, 39 Santa Catalina.

\begin{tabular}{|c|c|}
\hline Taxones infragenéricos & $\begin{array}{l}\text { Distritos de los subsectores de Cuba Oriental y Cuba Occidental } \\
\text { (bipolaridad) }\end{array}$ \\
\hline Eugenia rigidula Britton \& P.Wilson & $6,27,28,33,36,37,39$ \\
\hline Eugenia scaphophylla C.Wright & $6,32,33,37,39$ \\
\hline Lisianthius glandulosus A.Rich. & $5,6,27,28,29,31,32,33,35,37,39$ \\
\hline Mecranium haemanthum Triana & $6,29,32$ \\
\hline Pilea ovalifolia Britton \& P.Wilson & $6,28,29$ \\
\hline Psidium parvifolium Griseb. & $6,7,25,30,31,32,33,39$ \\
\hline Sapium adenodon Griseb. & $5,33,36,39$ \\
\hline \multirow[t]{2}{*}{ Tetrazygiopsis laxiflora (Naud.) Borhidi } & $6,28,29,33,35$ \\
\hline & $\begin{array}{c}\text { Distritos de los subsectores de Cuba Oriental y Cuba Central } \\
\text { (distribución bisectorial) }\end{array}$ \\
\hline Arthrostylidium urbanii Pilger & $15,16,19,20,21,22,24,31,32,33,35$ \\
\hline Banara glaberrima C.Wright ex Griseb. & 19,28 \\
\hline Callophyllum rivulare Bisse & $22,23,24,25,26,27,33,35,36,37$ \\
\hline Clusia tetrastigma Vesque & $21,28,29,32,33,36,39$ \\
\hline Connarus reticulatus Griseb. & $23,24,25,33,34$ \\
\hline Elytraria planifolia Leonard ssp. planifolia & $21,22,31$ \\
\hline Eugenia revoluta Berg. & $15,28,29,33,37,39$ \\
\hline Garcinia ruscifolia (Urb.) Borhidi & $14,21,23,31,32,33,37,39$ \\
\hline Plumeria cubensis Urb. & $14,15,19,21,22,23,25,26,31,33,36$ \\
\hline Phialanthus oblongatus Urb. & $23,31,33,39$ \\
\hline Psidium balium Urb. & $21,27,28$ \\
\hline Savia cuneifolia Urb. & $21,33,36,39$ \\
\hline $\begin{array}{l}\text { Spaniopappus hygrophilus (Alain) King \& } \\
\text { Robins. }\end{array}$ & $19,31,32,33,34,39$ \\
\hline Spirotecoma apiculata (Britton) Alain & $23,31,32,33,36,37$ \\
\hline Spirotecoma holguinensis (Britton) Alain & $23,24,31$ \\
\hline \multirow[t]{2}{*}{ Vernonia inaequiserrata Sch.Bip } & $19,20,26,28,29,30,31,35,36,39$ \\
\hline & $\begin{array}{c}\text { Distritos de los subsectores de Cuba Oriental, Cuba Central y } \\
\text { Cuba Occidental (distribución trisectorial) }\end{array}$ \\
\hline Ateramnus albicans (Griseb.) Rothm. & $2,5,6,14,22,24,26,32,33$ \\
\hline $\begin{array}{l}\text { Casearia sylvestris Sw. ssp. myricoides } \\
\text { (Griseb.) J.E.Gut. }\end{array}$ & $2,6,7,12,14,16,20,21,31,33$ \\
\hline Eugenia asperifolia Berg. & $6,9,13,16,20,24,26,27,33$ \\
\hline Eugenia heterophylla A.Rich. & $6,19,21,22,31,34$ \\
\hline Eugenia rimosa $\mathrm{C}$. Wright \& Sauvalle & $4,5,6,7,9,13,19,33,39$ \\
\hline Linodendron cubanum Griseb. & $10,21,28,32,33,37,39$ \\
\hline Mikania ranunculifolia A.Rich. & $2,6,7,8,13,14,16,19,22,28,29$ \\
\hline Myrica punctata Griseb. & $12,19,26,28,29,31,33,39$ \\
\hline
\end{tabular}


Gayana Bot. 66(2), 2009

Continuación Tabla V

Pimenta filipes (Urb.) Burret

$5,13,24,32,33,36$

Sapium daphnoides Griseb.

$6,10,17,19,28,29$

Psychotria lasiophtalma Griseb.

$6,19,27,28,29,33,39$

Tabebuia simplicifolia Carabia ex Alain

$7,14,31,32,33,39$

Vernonia gnaphaliifolia A.Rich.

$4,6,13,14,15,16,22,26,28$

Wallenia bumelioides (Griseb.) Mez

$1,6,7,14,21,24,28$

Xylopia obtusifolia (A.DC.) A.Rich.

$1,7,10,14,15,17,24,26,34$

ANÁLISIS DE LA DISTRIBUCIÓN DE LAS ESPECIES NO ENDÉMICAS

Las espermatofitas no endémicas mostraron mayor afinidad florística con el resto del Reino Neotropical y dentro de éste las relaciones fueron más estrechas con la Región Caribe, cuando se comparó con la Brasiliana y la Andina. Con el Reino Paleotropical el porcentaje de taxones infragenéricos compartidos fue menor, no hubo casi diferencias entre la Región África-India y la Indomalaya. En el Holártico, sobre todo en el Neártico, se detectó una mayor cantidad de especies compartidas en comparación con el reino anterior y aquí las relaciones fueron más estrechas con los elementos que provienen del sur y el sureste de los Estados Unidos de América. Las afinidades con Australia resultaron muy débiles (Tablas VI y VII).

TaBLA VI. Afinidades florísticas de las pluvisilvas del Subsector Nor-Oriental.

TABLE VI. Floristic relationships of rainforest from North-Oriental Sub-sector.

\begin{tabular}{lcc}
\hline \multicolumn{1}{c}{ Geoelementos } & $\begin{array}{c}\mathrm{N}^{\circ} \text { taxones } \\
\text { infragenéricos }\end{array}$ & Porcentaje \\
\hline REGión CARIBE & & 27 \\
SuBREGIÓN ANTILLANA & 191 & 21 \\
Española & 147 & 21 \\
Jamaica & 143 & 16 \\
Puerto Rico & 114 & 13 \\
Antillas Menores & 89 & \\
Sur de la Florida, Bahamas y Bermudas & & 15 \\
SuBREGIÓN MÉXICo-VENEZUELA & 107 & 9 \\
Guatemala-Panamá & 60 & 11 \\
Norte de Venezuela-Colombia & 75 & 12 \\
Baja California-México & 81 & 10 \\
Región Brasiliana & 69 & 1 \\
Región Andina & 6 & 1 \\
Región África-India & 8 & 2 \\
Región Indomalaya & 16 & 0 \\
Canadá-EUA-N. de México & 1 & 1 \\
Eurasia & 4 & \\
Australia & & \\
\hline
\end{tabular}


Fitogeografía de las pluvisilvas de Cuba Oriental: Martínez-Quesada, E.

TABLA VII. Afinidades florísticas de las pluvisilvas del Subsector Sur-Oriental.

TABLE VII. Floristic relationships of rainforest from South-Oriental Sub-sector.

\begin{tabular}{|c|c|c|}
\hline Geoelementos & $\begin{array}{c}\mathrm{N}^{\mathrm{o}} \text { taxones } \\
\text { infragenéricos }\end{array}$ & Porcentaje \\
\hline \multicolumn{3}{|l|}{ REGIÓN CARIBE } \\
\hline \multicolumn{3}{|l|}{ SUBREGIÓN ANTILLANA } \\
\hline Española & 212 & 48 \\
\hline Jamaica & 188 & 42 \\
\hline Puerto Rico & 176 & 40 \\
\hline Antillas Menores & 130 & 29 \\
\hline Sur de la Florida, Bahamas y Bermudas & 92 & 20 \\
\hline \multicolumn{3}{|l|}{ SuBREGIÓN MÉXICO- VeneZuela } \\
\hline Guatemala-Panamá & 123 & 28 \\
\hline Norte de Venezuela- Colombia & 81 & 18 \\
\hline Baja California-México & 98 & 22 \\
\hline Región Brasiliana & 108 & 24 \\
\hline Región Andina & 91 & 16 \\
\hline Región África-India & 10 & 2 \\
\hline Región Indomalaya & 9 & 2 \\
\hline Canadá-EUA-N de México & 18 & 4 \\
\hline Eurasia & 2 & 0 \\
\hline Australia & 6 & 1 \\
\hline
\end{tabular}

En el Caribe, la mayoría de los taxones infragenéricos compartidos correspondieron a la Subregión Antillana y dentro de ésta, las Antillas Mayores mostraron el porcentaje más elevado, principalmente La Española. También hubo una cantidad considerable de taxones infragenéricos que se compartieron con las Antillas Menores. En la Subregión México-Venezuela, las afinidades fueron más estrechas con Guatemala-Panamá (Tablas VI y VIII).

En los dendrogramas obtenidos se observaron dos grandes grupos que se separaron para conformar otros subgrupos que se interrelacionaron, aunque no de la misma forma en los subsectores NorOriental y Sur-Oriental (Figs. 4 y 5).

En el Subsector Nor-Oriental se observó que uno de estos grupos estuvo conformado en su mayor parte por las provincias florísticas de la Subregión Antillana, en el que a su vez se evidenció la estrecha relación entre los subgrupos
Cuba y La Española y entre Puerto Rico y las Antillas Menores, del que se separó Jamaica. Por otra parte, la provincia Sur de la Florida, Bahamas y Bermudas estuvo relacionada con las provincias de la Subregión México-Venezuela, aunque se aisló conjuntamente con el Norte de Venezuela y Colombia del subgrupo que conformaron Baja California-México y Guatemala-Panamá.

En el Subsector Sur Oriental se obtuvo un resultado distinto en cuanto al agrupamiento de las provincias florísticas. En un primer grupo hubo una mayor afinidad entre el subgrupo Cuba y La Española, del que se segregó Jamaica. Sin embargo, en el otro grupo se detectó a Puerto Rico y las Antillas Menores que se separaron a partir de un subgrupo conformado por las provincias de la subregión México-Venezuela y el Sur de la Florida, Bahamas y Bermudas. En este caso la provincia Baja California-México se encontró separada de las provincias Guatemala-Panamá y Norte de Venezuela y Colombia. 
Gayana Bot. 66(2), 2009

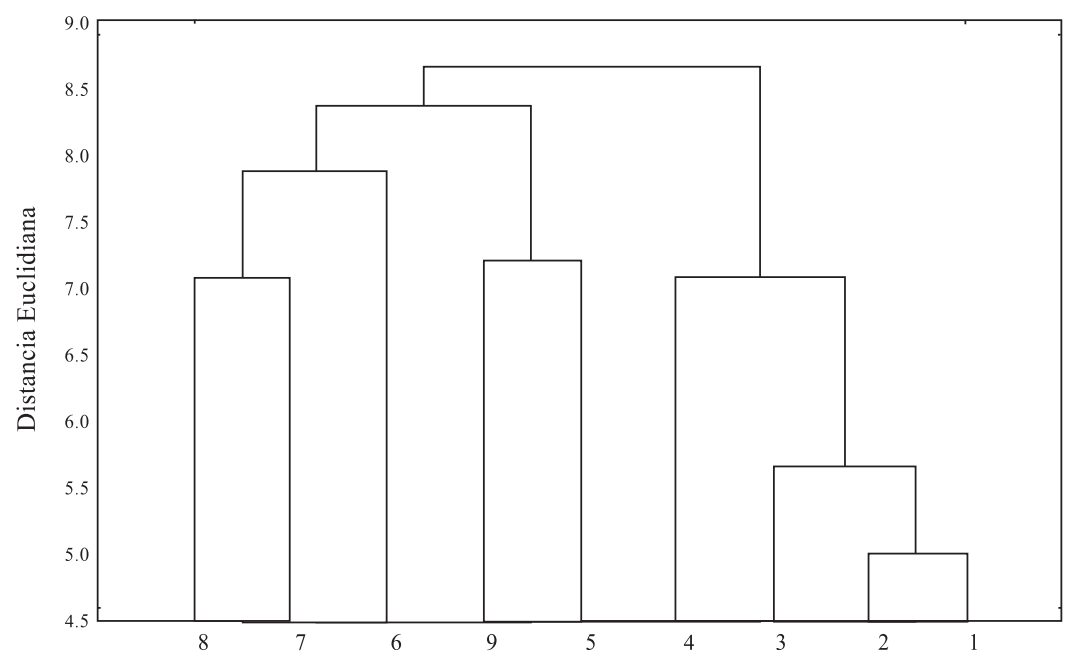

Figura 4. Dendrograma que muestra las relaciones de afinidad entre las espermatofitas de las pluvisilvas del Subsector Nor-Oriental y la Región Caribe.

FIGURE 4. Dendrogram showing the relationships between spermatophytes from rainforest of North-Oriental Sub-sector and Caribbean Region.

1. Baja California-México, 2. Guatemala-Panamá, 3. Venezuela-Colombia Norte, 4. Sur de la Florida-BahamasBermudas, 5. Española, 6. Jamaica, 7. Puerto Rico, 8. Antillas Menores, 9. Cuba.

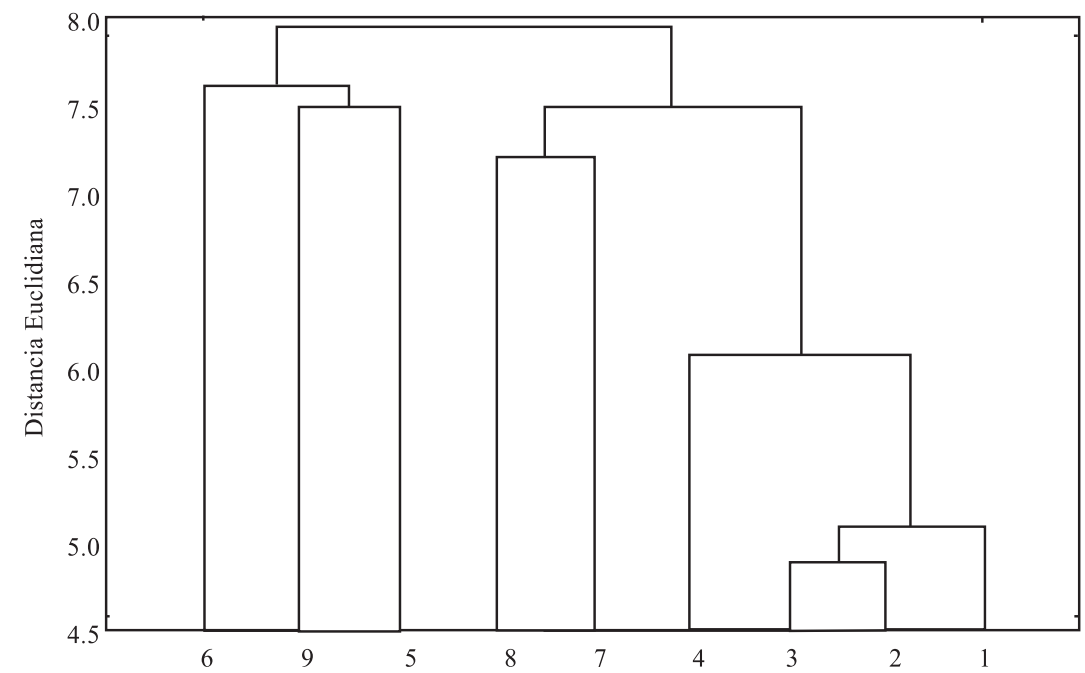

FIGURA 5. Dendrograma que muestra las relaciones de afinidad entre las espermatofitas de las pluvisilvas del Subsector Sur-Oriental y la Región Caribe.

FigURE 5. Dendrogram showing the relationships between spermatophytes from rainforest of South-Oriental Sub-sector and Caribbean Region.

1. Baja California-México, 2. Guatemala-Panamá, 3. Venezuela-Colombia Norte, 4. Sur de la Florida-BahamasBermudas, 5. Española, 6. Jamaica, 7. Puerto Rico, 8. Antillas Menores, 9. Cuba. 
Fitogeografía de las pluvisilvas de Cuba Oriental: Martínez-Quesada, E.

ANÁlisis DE LOS CENTROS DE ORIGEN Y EVOLUCIÓN DE LAS FAMILIAS

La mayor parte de las familias, cuyo origen se conoció con certeza, provinieron de Gondwana (61) y el resto de Laurasia (28). Un total de 15 correspondieron a las de origen incierto.

En Gondwana, las familias que procedieron del Centro Amazónico representaron la mayoría (31) y luego le siguieron las del centro Andes del Norte (22). Por otra parte, se observaron pocas familias cuyo origen proviene de los Andes del Sur (cinco) y de las Áreas secas de Gondwana (tres) (Tabla VIII).
Al nivel de especie los resultados son casi iguales, pero el Centro Andes del Norte superó al Amazónicoporelnúmerodetaxonesinfragenéricos que presentó (450 y 225, respectivamente). En este caso hubo una fuerte influencia de las familias Orchidaceae, Rubiaceae, Melastomataceae y Asteraceae por la gran cantidad de especies que acumularon (68\%). En el centro Amazónico se destacó Euphorbiaceae (21\%). Por su parte, el centro Andes del Sur también fue importante y las especies procedieron en lo fundamental de Myrtaceae (74\%) (Tabla VIII).

TABLA VIII. Familias, número de géneros y especies hallados en las pluvisilvas de la Región Oriental de Cuba.

TABLE VIII. Families, number of genera and species found on rainforest of Oriental Region of Cuba.

\begin{tabular}{|c|c|c|c|}
\hline Familia & Centros de origen y evolución & $\mathrm{N}^{\circ}$ géneros & $\mathrm{N}^{\circ}$ especies \\
\hline Acanthaceae & Andes del Norte & 3 & 3 \\
\hline Agavaceae & Laurasia & 2 & 2 \\
\hline Amaranthaceae & Origen incierto & 1 & 1 \\
\hline Anacardiaceae & Amazonía & 2 & 2 \\
\hline Annonaceae & Amazonía & 4 & 8 \\
\hline Apocynaceae & Amazonía & 9 & 13 \\
\hline Aquifoliaceae & Laurasia & 1 & 10 \\
\hline Araceae & Andes del Norte & 2 & 5 \\
\hline Araliaceae & Andes del Norte & 2 & 2 \\
\hline Arecaceae & Amazonía & 4 & 5 \\
\hline Aristolochiaceae & Laurasia & 1 & 2 \\
\hline Asclepiadaceae & Origen incierto & 4 & 6 \\
\hline Asteraceae & Andes del Norte & 23 & 59 \\
\hline Begoniaceae & Andes del Norte & 1 & 3 \\
\hline Bignoniaceae & Amazonía & 5 & 16 \\
\hline Bombacaceae & Amazonía & 1 & 1 \\
\hline Boraginaceae & Origen dudoso & 4 & 8 \\
\hline Bromeliaceae & Andes del Norte & 6 & 19 \\
\hline Bruneliaceae & Andes del Norte & 1 & 1 \\
\hline Burmanniaceae & Guyana & 2 & 2 \\
\hline Burseraceae & Origen dudoso & 1 & 4 \\
\hline Buxaceae & Laurasia & 1 & 6 \\
\hline Cactaceae & Áreas secas de Gondwana & 1 & 1 \\
\hline Campanulaceae & Andes del Norte & 2 & 6 \\
\hline Canellaceae & Origen incierto & 1 & 1 \\
\hline Caprifoliaceae & Laurasia & 1 & 1 \\
\hline Cecropiaceae & & 1 & 1 \\
\hline Celastraceae & Laurasia & 3 & 7 \\
\hline Chlorantaceae & Laurasia & 1 & 4 \\
\hline Chrysobalanaceae & & 1 & 1 \\
\hline Cleomaceae & Áreas secas de Gondwana & 1 & 1 \\
\hline
\end{tabular}


Gayana Bot. 66(2), 2009

\begin{tabular}{|c|c|c|c|}
\hline Familia & Centros de origen y evolución & No. géneros & No. especies \\
\hline Clethraceae & Laurasia & 1 & 1 \\
\hline Clusiaceae & Andes del Norte & 4 & 16 \\
\hline Combretaceae & Amazonía & 2 & 3 \\
\hline Connaraceae & Amazonía & 1 & 1 \\
\hline Convolvulaceae & Amazonía & 1 & 1 \\
\hline Cucurbitaceae & Origen incierto & 2 & 3 \\
\hline Cunnoniaceae & Andes del Sur & 1 & 1 \\
\hline Cupressaceae & Andes del Sur & 1 & 1 \\
\hline Cyperaceae & Origen incierto & 3 & 18 \\
\hline Cyrillaceae & Laurasia & 2 & 8 \\
\hline Dichapetalaceae & Amazonía & 1 & 2 \\
\hline Dilleniaceae & Amazonía & 2 & 2 \\
\hline Dioscoreaceae & Origen incierto & 2 & 6 \\
\hline Droseraceae & Laurasia & 1 & 1 \\
\hline Ebenaceae & Amazonía & 1 & 1 \\
\hline Elaeocarpaceae & Amazonía & 1 & 1 \\
\hline Ericaceae & Andes del Norte & 2 & 15 \\
\hline Eriocaulaceae & Origen incierto & 1 & 1 \\
\hline Erythroxylaceae & Áreas secas de Gondwana & 1 & 2 \\
\hline Euphorbiaceae & Amazonía & 18 & 47 \\
\hline Fabaceae & Amazonía & 15 & 28 \\
\hline Flacourtiaceae & Amazonía & 7 & 16 \\
\hline Garryaceae & Laurasia & 1 & 1 \\
\hline Gentianaceae & Laurasia & 5 & 7 \\
\hline Gesneriaceae & Andes del Norte & 4 & 15 \\
\hline Goodeniaceae & Origen incierto & 1 & 1 \\
\hline Heliconiaceae & Andes del Norte & 1 & 1 \\
\hline Hyppocrateaceae & Amazonía & 2 & 2 \\
\hline Hypericaceae & & 1 & 4 \\
\hline Illiciaceae & Laurasia & 1 & 1 \\
\hline Lamiaceae & Origen dudoso & 2 & 2 \\
\hline Lauraceae & Amazonía & 5 & 15 \\
\hline Lentibulariaceae & Origen incierto & 2 & 3 \\
\hline Linaceae & Origen dudoso & 1 & 1 \\
\hline Loranthaceae & Andes del Norte & 1 & 1 \\
\hline Lythraceae & Laurasia & 1 & 3 \\
\hline Magnoliaceae & Amazonía & 2 & 7 \\
\hline Malpighiaceae & Amazonía & 6 & 16 \\
\hline Marcgraviaceae & Andes del Norte & 1 & 3 \\
\hline Mayacaceae & Guyana & 1 & 1 \\
\hline Melastomataceae & Andes del Norte & 15 & 62 \\
\hline Meliaceae & Amazonía & 4 & 4 \\
\hline Menispermaceae & Amazonía & 2 & 3 \\
\hline Moraceae & Amazonía & 4 & 5 \\
\hline Myricaceae & Laurasia & 1 & 4 \\
\hline Myrsinaceae & Andes del Norte & 3 & 11 \\
\hline Myrtaceae & Andes del Sur & 12 & 42 \\
\hline Nyctaginaceae & Andes del Norte & 2 & 3 \\
\hline Ochnaceae & Amazonía & 1 & 2 \\
\hline
\end{tabular}


Fitogeografía de las pluvisilvas de Cuba Oriental: Martínez-Quesada, E.

\begin{tabular}{|c|c|c|c|}
\hline Familia & Centros de origen y evolución & No. géneros & No. especies \\
\hline Olacaceae & Amazonía & 1 & 2 \\
\hline Oleaceae & Laurasia & 2 & 4 \\
\hline Orchidaceae & Andes del Norte & 49 & 110 \\
\hline Oxalidaceae & Andes del Norte & 1 & 1 \\
\hline Papaveraceae & Laurasia & 1 & 1 \\
\hline Passifloraceae & Andes del Norte & 1 & 6 \\
\hline Phytolaccaceae & Origen incierto & 2 & 2 \\
\hline Picramniaceae & Amazonía & 1 & 1 \\
\hline Pinaceae & Laurasia & 1 & 2 \\
\hline Piperaceae & Andes del Norte & 3 & 31 \\
\hline Poaceae & Origen incierto & 16 & 34 \\
\hline Podocarpaceae & Andes del Sur & 1 & 1 \\
\hline Polygalaceae & Amazonía & 1 & 1 \\
\hline Polygonaceae & Origen incierto & 1 & 14 \\
\hline Rhamnaceae & Laurasia & 6 & 7 \\
\hline Rhyzophoraceae & Amazonía & 1 & 1 \\
\hline Rosaceae & Laurasia & 2 & 3 \\
\hline Rubiaceae & Andes del Norte & 26 & 76 \\
\hline Rutaceae & Origen incierto & 5 & 10 \\
\hline Sabiaceae & Origen dudoso & 1 & 1 \\
\hline Sapindaceae & Amazonía & 6 & 10 \\
\hline Sapotaceae & Amazonía & 5 & 13 \\
\hline Simaroubaceae & Amazonía & 1 & 1 \\
\hline Smilacaceae & Laurasia & 1 & 3 \\
\hline Solanaceae & Andes del Sur & 4 & 12 \\
\hline Staphyleaceae & Laurasia & 1 & 1 \\
\hline Styracaceae & Laurasia & 1 & 1 \\
\hline Symplocaceae & Laurasia & 1 & 4 \\
\hline Theophrastaceae & Laurasia & 1 & 3 \\
\hline Theaceae & Laurasia & 5 & 11 \\
\hline Thymeleaceae & Origen incierto & 2 & 3 \\
\hline Turneraceae & Origen dudoso & 1 & 1 \\
\hline Ulmaceae & Laurasia & 1 & 3 \\
\hline Urticaceae & Origen dudoso & 4 & 13 \\
\hline Verbenaceae & Origen incierto & 4 & 11 \\
\hline Viscaceae & & 2 & 11 \\
\hline Vitaceae & Laurasia & 2 & 6 \\
\hline
\end{tabular}

El análisis de los centros de origen de las familias por tipos de pluvisilvas mostró diferencias entre ellas (Tabla IX). En la Pluvisilva submontana sobre suelos de mal drenaje, la Pluvisilva de baja altitud y submontana sobre ofiolitas, la Pluvisilva de baja altitud sobre complejo metamórfico y la Pluvisilva montana las familias del centro Amazónico se observaron en mayor cantidad; sin embargo, las norandinas superaron a las amazónicas en la Pluvisilva submontana sobre complejo metamórfico, aunque las diferencias no fueron tan grandes. Por otra parte, en la Pluvisilva montana y la Pluvisilva de baja altitud y submontana sobre ofiolitas las que procedieron de Laurasia superaron en número al resto de ellas; mientras que en la segunda se igualaron a las del centro Amazónico. Este último centro fue el más importante dentro de Gondwana, excepto en la Pluvisilva submontana sobre complejo metamórfico. 
Gayana Bot. 66(2), 2009

TABLA IX. Cantidad de familias por centros de origen en las pluvisilvas.

TABLE IX. Amount of families by evolutionary centers on rainforest types.

A. Pluvisilva de baja altitud y submontana sobre ofiolitas, B. Pluvisilva submontana sobre suelos de mal drenaje, C. Pluvisilva de baja altitud sobre complejo metamórfico, D. Pluvisilva submontana sobre complejo metamórfico, E. Pluvisilva montana.

\begin{tabular}{lccccc}
\hline \multicolumn{1}{c}{ Centros de origen y evolución de las familias } & \multicolumn{3}{c}{ Tipos de pluvisilvas/ $\mathrm{N}^{\mathbf{2}}$ de familias } \\
& $\mathrm{A}$ & $\mathrm{B}$ & $\mathrm{C}$ & $\mathrm{D}$ & $\mathrm{E}$ \\
\hline Andes del Norte & 20 & 15 & 12 & 15 & 19 \\
Andes del Sur & 4 & 4 & 1 & 1 & 4 \\
Áreas secas de Gondwana & 1 & 1 & 1 & 0 & 1 \\
Amazónicas & 22 & 21 & 14 & 13 & 20 \\
Guyana & 1 & 2 & 1 & 0 & 0 \\
Laurasia & 22 & 17 & 5 & 5 & 23 \\
Origen incierto & 9 & 10 & 6 & 3 & 9 \\
\hline
\end{tabular}

\section{DISCUSION}

El hecho de que la mayoría de los endemismos estén ubicados en el Sector Cuba Oriental (predominio de los sectoriales) se relaciona directamente con su historia paleogeográfica, como expusieron Reyes (1994) y López (1998a) y con los factores ecológicos que determinan la gran acumulación de las especies endémicas en las subregiones Grupo Orográfico Sagua-Baracoa y Sierra Maestra, lo que coincide con lo señalado por López et al. (1994a, 1994b). Estos factores fueron decisivos en la especiación, al punto de que la mayor cantidad de las especies que habitaron las pluvisilvas permanecieron en ese sector y un menor número se extendió hasta Cuba Central y Cuba Occidental durante las migraciones internas; o quizás la cantidad de especies que migró hacia otros sectores no fue tan baja, pero no muchas lograron sobrevivir durante las extinciones masivas causadas por la falta de refugios necesarios durante las transgresiones marinas y los cambios climáticos, como indicaron Ortega \& Arcia (1982). De acuerdo con López (1998b) el distrito Serpentinitas de MoaToa-Baracoa debió haber funcionado como un centro de radiación adaptativa, en el que después de cada evento catastrófico los taxones que lograron refugiarse formaron nuevas especies en condiciones de aislamiento.

Por otra parte, la elevada cantidad de endemismos multidistritales orientales demuestra que la mayoría de las especies se distribuyeron por Cuba Oriental, donde abarcaron un área más grande que las que especiaron en una más reducida, por lo que de acuerdo con López et al. (1994a) hubo un intercambio amplio de germoplasma en esta región.

La gran acumulación de endemismos hallados en las pluvisilvas del distrito Serpentinitas de Moa-Toa-Baracoa se justifica por la combinación de varios factores esenciales (relieve montañoso, sustrato ofiolítico y abundantes precipitaciones), así como también el prolongado período de aislamiento que Cuba Oriental tuvo durante su formación y el tiempo que Sagua-Baracoa lleva emergido como sistema montañoso de forma estable, de acuerdo con López et al. (1994b), Reyes (2000) y Coleman \& Alexander (2004). Otros distritos con alguna importancia por la presencia de endemismos y que se relacionan con Serpentinitas de Moa-ToaBaracoa, aunque no de forma estrecha, son Sierra de Nipe y Sierra de Cristal. En ellos las pluvisilvas se hallan bajo condiciones similares de altitud, sustrato y precipitaciones. Además, se coincide con López et al. (1994b) en el hecho de que la cercanía geográfica entre ambos macizos montañosos pudo haber permitido un intercambio florístico por un tiempo prolongado. No obstante, los procesos de especiación y la florogénesis orófila de las sierras de Nipe y de Cristal parecen haber tenido un desarrollo separado al de Serpentinitas de Moa-Toa-Baracoa, debido a la gran cantidad de endemismos estrictos presentes en este último que no se observan en ellos. También, en el Eoceno medio ya estaban separados los bloques Nipe-Cristal de Moa-Baracoa-Sierra del Purial-Asunción. 
Fitogeografía de las pluvisilvas de Cuba Oriental: Martínez-Quesada, E.

Respecto a la Pluvisilva montana, ubicada en el distrito montañoso de la Cordillera del Turquino por encima de los 800 ó $900 \mathrm{~m}$ snm, la acumulación de endemismos distritales se debe al factor altitudinal y a que está expuesta a otros factores como abundantes precipitaciones, elevados valores de humedad relativa, alta influencia de las precipitaciones horizontales y baja evaporación (Viña 2005). Todos ellos se compensan o refuerzan mutuamente, lo que permite el desarrollo de las especies, principalmente las epífitas. En este caso se destaca Orchidaceae y dentro de ésta, los géneros Pleurothallis y Lepanthes. López et al. (1994a) señalaron que no existe otra parte de Cuba donde se hallen tantas orquídeas endémicas y Hespenheide (1973) indicó que en ningún lugar del territorio cubano Lepanthes está mejor representado. Sin embargo, como se expuso anteriormente, los taxones infragenéricos con hábito epífito, aunque resaltan en esta pluvisilva, no son dominantes en ninguno de los bosques estudiados y por otra parte son pocas las orquídeas endémicas halladas.

En la Pluvisilva montana de Gran Piedra, donde existe una altitud por encima de los 1.000 m s.n.m. y condiciones climáticas y de sustrato similares a las halladas en el distrito montañoso de la Cordillera del Turquino, el número de endemismos es muy bajo; incluso los distritales y esto hace que en el dendrograma se separe del resto de los distritos en Cuba Oriental. Ningún autor, excepto López et al. (1994a), trató de explicar la causa de este fenómeno. Ellos indicaron que pudiera estar relacionado con la reciente terminación de los procesos tectónicos que le dieron origen. No obstante, una causa lógica pudiera ser la ausencia del Bosque nublado, formación en la que según esos autores ocurrió la más grande especiación de epífitas cubanas. También, la extensión superficial que cubre el área comprendida entre los $1.000 \mathrm{~m} \mathrm{y}$ los $1.200 \mathrm{~m}$ de altitud es muy pequeña (menos de 5 $\mathrm{km}^{2}$ ) comparada con la de la Cordillera del Turquino a esta misma altura, y por otro lado, la Sierra de la Gran Piedra pudo haber funcionado como una zona de transición entre los macizos montañosos NipeSagua-Baracoa y la Sierra Maestra Occidental.

En Cuba Central las mayores relaciones son con el distrito Escambray. Según Formell (1989), Magaz (1989) y Portela et al. (1989) éste corresponde a un macizo montañoso considerado uno de los más complejos de Cuba, tanto por su geología como por su relieve y Díaz et al. (1986) señalaron que allí existen algunas de las unidades morfotectónicas más antiguas, lo que de acuerdo con López et al. (1994b) pudo haber facilitado la acumulación de endemismos durante un período prolongado. Aunque algunas especies compartidas entre las pluvisilvas de Cuba Oriental y Cuba Central se hallan en Escambray sobre ofiolitas, la mayoría se encuentra asociada al carso y forman parte de otros tipos de vegetación, como los bosques siempreverdes y el complejo de mogotes. Ello parece haber sido una consecuencia de la adaptación a este tipo de sustrato de los elementos montanos que migraron de este a oeste, incluso esto también lo mencionaron Fernández et al. (1985) al analizar la distribución de las Rubiáceas endémicas cubanas. Borhidi $(1991,1996)$ indicó que las montañas de Sagua-Baracoa, en el Subsector Nor Oriental, constituyen el principal centro evolutivo desde donde partieron las migraciones de los taxa orófilos hacia otros distritos de Cuba Oriental. Por otra parte, Reyes et al. (1991) señalaron que en este primer estadio pudo haber existido una conexión entre dicha subregión y Cuba Central a través de Guamuhaya. Si se tiene en cuenta que fuera de Cuba Oriental este sistema montañoso es el único que posee alturas superiores a los 1.000 $\mathrm{m}$ se infiere que las partes más elevadas pudieron servir como refugios durante las transgresiones marinas que mencionaron Raven \& Axelrod (1974) y los períodos fríos del Pleistoceno, donde según Acevedo (1986) la sequedad del clima pudo haber inducido a que la flora se retrajera a las partes más altas, lo que probablemente posibilitó que muchas especies compartidas de ambos sectores sobrevivieran a este fenómeno.

El otro distrito que presenta afinidades florísticas con las pluvisilvas de Cuba Oriental es Sierra del Rosario, en Cuba Occidental, aunque de acuerdo con López et al. (1994a) éstas se observan fundamentalmente con especies de Bosques siempreverdes. Según Iturralde-Vinent (1982) la Sierra del Rosario se considera uno de los biótopos más antiguos del archipiélago cubano y es probable que no quedara sumergida de forma total durante las transgresiones marinas (López et al. 1985). También Ortega \& Arcia (1982) señalaron que en esta zona el clima se ha mantenido bastante estable desde el Terciario y probablemente llovió más durante los largos períodos secos del Pleistoceno, lo cual favoreció la perdurabilidad de la flora orófila. 
La distribución de las especies endémicas bisectoriales presentes en las pluvisilvas orientales se corresponde con aquellas que llegan hasta Cuba Central, donde se acumulan fundamentalmente en el distrito Escambray. Se conoce que el distrito Serpentinitas de Moa-Toa-Baracoa es el principal centro de especiación en Cuba y que fungió como una fuente para los núcleos cercanos (Borhidi 1991, Reyes et al. 2000), entre ellos la Sierra Maestra, y que este último pudo haber funcionado de igual forma para determinados taxones, a partir del cual ocurrieron migraciones hacia el primero (López et al. 1994a). Esto permite afirmar que aunque la mayor parte de las especies compartidas con Cuba Central se hallan en el Subsector NorOriental, hay también una gran influencia del Sur-Oriental. Por otra parte, los contactos entre la parte oriental y central de protoCuba permitieron el intercambio entre los elementos montanos que durante la florogénesis ocuparon áreas con diversas condiciones ambientales, como las que se observan en la actualidad en esos macizos montañosos y que pudieron sobrevivir refugiados en las partes más altas (López et al. 1994b).

Lo anteriormente explicado también se evidencia entre las especies de distribución trisectorial que se encuentran en Cuba Oriental, Cuba Central y el Subsector Pinar del Río, en la que los taxones infragenéricos se comparten principalmente con Escambray y Sierra del Rosario, donde se hallan las mayores alturas fuera de Cuba Oriental. Esto apoya la idea de López et al. (1994b) de que en Cuba existe una disyunción orófila, que de acuerdo con Bisse (1980) se produjo debido a los cambios climáticos.

La menor cantidad de especies con distribución bipolar indica que fueron pocos los elementos que durante la migración este-oeste se establecieron en Cuba Occidental, lo que de acuerdo con López et al. (1994b) puede estar relacionado con la distancia entre ambas partes del territorio que funciona como una barrera de aislamiento para las especies; también el sustrato puede influir, sobre todo para los taxones infragenéricos que se establecen en el complejo ofiolítico en cada uno de los sectores.

Las especies no endémicas de las pluvisilvas de Cuba Oriental se distribuyen mayoritariamente en el Reino Neotropical, lo que de acuerdo con Gentry (1982) presenta una relación estrecha con los procesos de intercambios florísticos que tuvieron lugar entre los dos supercontinentes Laurasia y
Gondwana, cuando las Proto-Antillas sirvieron para ello. Según Gentry (1982) e Iturralde-Vinent (1988), los eventos que condicionaron la formación de Cuba como archipiélago y los sucesivos movimientos de la flora desde ésta hacia el continente americano y viceversa duraron mucho más tiempo que la separación de África y los demás miembros de Gondwana, durante su desmembramiento. Esto justifica las relaciones más débiles con las regiones África-India, Indomalaya y el Reino Australiano, con los que hubo menos intercambio y explica también aquéllas relativamente más estrechas con el Neártico, fundamentalmente con los elementos que provienen del sur y el sureste de los Estados Unidos de América. Se conoce que el flujo de biodiversidad entre Laurasia y Gondwana es muy antiguo, probablemente desde el Paleozoico Superior; momento en el que Odreman \& Ricardi (1990) señalaron una flora equivalente entre México y Brasil-Argentina, así como entre Venezuela y Laurasia, respectivamente. Las migraciones principalmente tuvieron lugar a través de la Dorsal Caimán para Cuba Oriental, en el Cretácico. También Chiappy et al. (1988) expusieron una ruta migratoria entre Norteamérica y la costa norte de la parte centro-oriental de Cuba a través del banco carbonatado de las Bahamas que parece haber fluctuado de forma estable en su régimen de ascensos y descensos desde el Jurásico y por la que pudo ocurrir el paso de los elementos florísticos.

La mayor parte de las especies que forman parte de las pluvisilvas están compartidas con la Región Caribe, lo que demuestra que Cuba durante su formación estuvo más tiempo relacionada con las tierras que hoy conforman la cuenca de dicha región. Según Hernández (1989), durante el Cretácico Tardío se formó la Cordillera CaimánSierra Maestra, la que posteriormente se fracturó en el Eoceno Tardío. Eso pudo haber facilitado el intercambio florístico con el norte de América Central mientras existió la conexión, incluso Reyes (1994) y Reyes et al. (2000) expusieron que éste pudo continuar después de la ruptura por la cercanía al noreste de Cuba Oriental y la Sierra Maestra. Otra vía importante de intercambio, señalada por Iturralde-Vinent (1988), Borhidi (1991), Reyes et al. (1991) y Reyes (1994) fue la conexión entre Centroamérica y Cuba Oriental a través de La Española y Jamaica la que probablemente se realizó a través de una guirnalda de islas.

La relación florística más cercana que se 
Fitogeografía de las pluvisilvas de Cuba Oriental: Martínez-Quesada, E.

evidencia con La Española, Jamaica y Puerto Rico, se debe a que partes de sus territorios estuvieron conectadas en algún momento durante mucho tiempo, lo que posibilitó un mayor intercambio de especies. Se conoce que las rocas metamórficas mesozoicas de la parte oriental de Cuba y las de la Cordillera Septentrional de la República Dominicana constituyen fragmentos del Arco Cretácico Antillano. Además, HernándezSarlabous (1993) se refirió a la similitud geológica y petrológica que existe entre estas rocas cubanas y sus representantes en La Española, Jamaica y Puerto Rico. Otro ejemplo que justifica la antigüedad de esta conexión es la correlación hecha por Hernández y Canedo (1995) entre las ofiolitas de la Sierra del Convento en la provincia Guantánamo y el complejo Río San Juan que aflora en Puerto Plata (República Dominicana), las que debieron estar emergidas desde el Cretácico (Reyes et al. 2000). Esa estrecha afinidad florística también se puede observar en los dendrogramas obtenidos, donde Jamaica está más cerca en su relación con Cuba y La Española en el Subsector Sur Oriental; mientras que en el Nor Oriental tiene mayor relación con Puerto Rico y las Antillas Menores.

Según Rosen (1985) la unión de Jamaica con La Española, Cuba y la América Central (Yucatán) duró poco, pues ésta conjuntamente con las Islas Caimán constituyeron partes aisladas durante el mayor tiempo de la historia geológica del Caribe, en la que Raven \& Axelrod (1974) manifestaron que Jamaica pudo haber estado sumergida desde el Eoceno hasta el Mioceno debido a las múltiples transgresiones marinas. Según Iturralde-Vinent $(1996,1998)$ en el Eoceno Medio dominaron los depósitos de calizas, tanto en condiciones de aguas profundas como someras, lo que sugiere la limitada presencia de tierras emergidas, incluso Robinson (1976) y Robinson et al. (1977) expusieron que el grupo de calizas blancas de Jamaica central estuvo continuamente sumergido.

Por otra parte, Iturralde-Vinent \& MacPhee (1999) e Iturralde Vinent (2004) señalaron que las Montañas Azules estaban emergidas desde el Eoceno en una posición más al noreste de la actual y que se desplazaron con el movimiento de la Placa Caribe, por lo que parece ser la parte emergida más antigua de Jamaica. Según Iturralde-Vinent (1996) existen algunas semejanzas entre las rocas ofiolíticas y metavolcánicas de Cuba Oriental y las Montañas Azules, lo que demuestra que estos terrenos pertenecen a la misma provincia geológica y que debieron haber estado conectados en algún momento.

Sin embargo, la mayor cantidad de especies compartidas entre las pluvisilvas de Cuba Oriental y La Española denota un intercambio más prolongado en el tiempo. Areces (1985) ubica esta conexión desde el Eoceno Medio hasta el Oligoceno, momento en que el noroeste de La Española estaba unido al bloque hórstico del noreste de Cuba Oriental y muy próximo al sur de Oriente, aunque Oro (1989) señaló que éste pudo haber sido desde el Cretácico. También Cobiella (1983) expuso que al sur del archipiélago oriental existió una cadena montañosa (la Tierra de Bartlett) que debió haber facilitado el intercambio florístico entre ambos territorios, incluso Iturralde-Vinent (1988) señaló que su prolongación pudo haber involucrado a Jamaica.

La relación con la Antillas Menores es menos estrecha, aunque en Cuba Oriental tiene más importancia que en otros sectores fitogeográficos. Samek (1973) señaló que la afinidad florística de Cuba con esas islas es relativamente débil, pero el mismo autor reconoció que puede estar en dependencia de la región que se trate. Al observar los dendrogramas se aprecia que estas Antillas están más relacionadas con Puerto Rico que con cualquier otra isla del Caribe. Ello demuestra que los contactos con Cuba duraron menos tiempo y que al parecer el arco de las Antillas Menores estuvo más vinculado con Puerto Rico y la Dorsal Aves. Además, Roughgarden (1995) indicó que las rocas Jurásicas de la Désirade del archipiélago de Guadalupe poseen una composición mineralógica y química que se asemeja más a las de Puerto Rico que al resto de las Antillas Mayores. No obstante lo planteado, esas islas tuvieron una participación importante en la migración de las especies suramericanas hacia la Región Oriental, cuando ésta no tenía lugar a través de América Central.

La mayoría de las familias que existen en las pluvisilvas estudiadas proceden de Gondwana, lo que corrobora lo observado por Reyes (1994), Borhidi (1998) y López et al. (1994a, 1994b). En general las familias que proceden de Amazonía son las más numerosas, aunque se observan diferencias respecto al origen y evolución de éstas en cada tipo de pluvisilva, pero no tan grandes.

Según López et al. (1994b) las familias de origen Amazónico que contienen cualesquiera 
de los tipos de endemismos detectados en el distrito de Serpentinitas de Moa-Toa-Baracoa son predominantes; mientras que en la Cordillera del Turquino se da una situación similar con aquéllas donde se acumulan los endemismos totales y multisectoriales, pero en las que contienen las especies endémicas distritales predominan las Norandinas. Ello se entiende bien cuando se analiza que la mayor parte del endemismo estricto de este distrito se compone de epífitas, las que según Gentry (1982) y Gentry \& Dodson (1987) poseen la mayor riqueza florística en las familias de ese centro evolutivo. Por otra parte, aunque López et al. (1994a, 1994b) no establecen una relación entre los centros evolutivos de las familias y las formaciones vegetales, a no ser el Bosque nublado, se puede inferir que sus resultados corroboran lo encontrado en este estudio, ya que los bosques pluviales en Cuba Oriental ocupan la mayor extensión en los subsectores Nor-Oriental y SurOriental. También Reyes (1994) mencionó que en la flora fanerogámica cubana actual las familias de origen Amazónico se encuentran en superioridad cuando se comparan con las que tienen su origen en los Andes del Norte.

El otro grupo evolutivo de importancia es el Norandino, lo que se corresponde con lo señalado por López et al. (1994a, 1994b), Fagilde (2000a) y Reyes (1994) en relación a su incidencia en la flora cubana. Según Borhidi (1995), en las Antillas hay más familias Andinas que Amazónicas, pero cuando se comparan estas últimas con las Norandinas, en sus propios resultados, las que provienen de Amazonía son dominantes en nuestra flora. Al nivel de especie los Andes del Norte adquieren mayor importancia principalmente por las familias Orchidaceae, Rubiaceae, Melastomataceae y Asteraceae. Ello indica la fuerte especiación que tuvo lugar a partir de los taxones del mismo, sobre todo en las tres últimas, donde se detectaron tantos endemismos en este estudio. Fernández et al. (1985) mediante el ejemplo de Rubiaceae se refirieron a este aspecto. Según Gentry $(1982,1985)$ y Gentry \& Dodson (1987) la mayor parte de las especies vegetales de Centroamérica, incluidas las endémicas, son de origen Norandino y éstos especian fácilmente en esa porción del continente, precisamente con la que Cuba Oriental tiene su más alta relación fuera de las Antillas. Por eso puede asegurarse de que estos dos centros evolutivos constituyeron la fuente principal para las migraciones desde América del Sur.
Por otro lado, se observa que las familias provenientes de Laurasia también se destacan en estos bosques, mucho más en la Pluvisilva montana. Ejemplo de ello lo constituyen Aquifoliaceae, Caprifoliaceae y Celastraceae, las que tienen la mayor parte de sus representantes en este tipo de bosque. Esto puede estar relacionado con los contactos que tuvieron lugar entre Cuba y otras partes del continente, mediante los cuales migraron los elementos de Norteamérica. Según López et al. (1994a) los taxones de este origen alcanzaron los mayores valores de Cuba Oriental entre los endemismos totales, multidistritales orientales y estrictos de la Cordillera del Turquino y por otra parte, si se tiene en cuenta el planteamiento de Howard (1968) sobre el hecho de que los elementos nórdicos son dominantes en las montañas altas, fenómeno que fue comprobado por Seifriz (1943) en Cuba, queda claro la influencia de este centro en los bosques pluviales.

\section{CONCLUSIONES}

El mayor porcentaje de las especies endémicas encontradas en las pluvisilvas son del Sector Cuba Oriental (sectoriales) y dentro de éstas abundan más las compartidas entre varios distritos (multidistritales). Los distritos Serpentinitas de Moa-Toa-Baracoa y Cordillera del Turquino acumulan la mayoría de los endemismos distritales.

Las relaciones florísticas más estrechas de los endemismos de las pluvisilvas de Cuba Oriental se establecen con los distritos Escambray en Cuba Central y Sierra del Rosario en Cuba Occidental; mientras que en las no endémicas son principalmente con La Española, en la Región Caribe.

La mayoría de las familias que forman parte de las pluvisilvas provienen de Gondwana, sobre todo de los centros Amazónico y Norandino.

\section{AGRADECIMIENTOS}

A Orlando J. Reyes, Rosalina Berazaín y Ludgarda González, por la revisión crítica del documento. A Antonio López, por su valiosa ayuda en facilitar la base de datos con la distribución de las especies endémicas cubanas. A. Juan C. Reyes, por su ayuda técnica en el procedimiento del programa 
Statistica 98 y a Ansel Font por la localización de las coordenadas geográficas de algunos sitios explorados, donde se desarrollan las pluvisilvas.

\section{BIBLIOGRAFIA}

AcEvedo, M. 1986. El Cuaternario en el Mediterráneo Americano y sus inmediaciones. Revista Tecnológica, Geología 16(2): 59-64.

Alain, Hno. 1953. Flora de Cuba 3. Dicotiledóneas: Malpighiaceae a Myrtaceae. Contribuciones Ocasionales Museo Historia Natural Colegio "De La Salle" 13. La Habana. 502 pp.

Alain, Hno. 1957. Flora de Cuba 4. Dicotiledóneas: Melastomataceae a Plantaginaceae. Contribuciones Ocasionales Museo Historia Natural Colegio "De La Salle" 16. La Habana. $556 \mathrm{pp}$.

Alain, Hno. 1958. La flora de Cuba: Sus principales características. $\mathrm{Su}$ origen probable. Revista Sociedad Cubana de Botánica 15: 36-59, 84-96.

Alain, Hno. 1964. Flora de Cuba 5. RubialesValeraniales-Cucurbitales-CampanulalesAsterales. Publicaciones Asociación Estudiantes Ciencias Biológicas, Universidad de La Habana. $362 \mathrm{pp}$.

Albert Puentes, D. 2005. Meliaceae. En: W. Greuter \& R. Rankin Rodríguez (eds.), Flora de la República de Cuba. Serie A, Plantas Vasculares. Fascículo 10(5). Ed. Gantner. Ruggell, Liechtenstein. $44 \mathrm{pp}$.

Albert Puentes, D. \& A. López Almirall. 1986. Distribución de las fanerógamas endémicas de Sierra Maestra. Reporte de Investigación del Instituto de Botánica 11: 1-27.

Areces, A. 1985. Las relaciones florísticas entre Cuba y La Española como resultado de la evolución geodinámica del borde Norte-Caribe. pp. 217243. En: Memorias del Primer Simposio de Botánica [2-5 julio], Tomo 3. La Habana.

Arias Granda, I. 1998. Araceae. En: H. Manitz \& A. Gutjahr (eds.), Flora de la República de Cuba. Serie A, Plantas Vasculares. Fascículo 1(1). Ed. Koeltz Scientific Books. Königstein. 46 pp.

Barreto Valdés, A. 1998. Las Leguminosas (Fabaceae) de Cuba, I. Subfamilia Caesalpinioideae. Collectanea Botanica 24: 6-148.

BÄSSler, M. 1998. Mimosaceae. En: Flora de la República de Cuba. Serie A, Plantas Vasculares. Fascículo 2. Ed. Koeltz Scientific Books. Königstein. 202 pp.

Berazaín ItURRAlde, R. 1992. Ericaceae. En: Flora de la República de Cuba. Fontqueria 35: 21-80.

Beyra Matos, A., P. Herrera Oliver \& O. Casas Mestre. 2002. Taxonomía y distribución del género Galactia P. Br. (Papilionaceae) en Cuba. Brenesia 57-58: 95-112.

Bisse, J. 1980. La subdivisión florística de la Región Nororiental de Cuba. Revista del Jardín
Botánico Nacional, Universidad de La Habana 1(1): 111-118.

BorHIDI, A. 1991. Phytogeography and Vegetation Ecology of Cuba. Akadémiai Kiadó, Budapest. $857 \mathrm{pp}$.

Borhidi, A. 1995. Es la flora de las Antillas de origen andino? Acta Botánica Academia de Ciencias Hungáricas 39(3-4): 201-216.

BorHIDI, A. 1996. Phytogeography and Vegetation Ecology of Cuba (2nd. ed.). Akademiai Kiadó, Budapest. $926 \mathrm{pp}$.

BorhidI, A. 1998. Fitogeografía y Ecología de la Vegetación de Cuba. Escuela Doctoral Botánica y Ciencias de la Vegetación, Janus Pannonius Univ., Pécs, Hungría. 56 pp.

BorhIDI, A. \& O. MuÑIZ. 1986. Phytogeographic survey of Cuba II. Floristic relationships and phytogeographic subdivision. Acta Botánica Academia de Ciencias Hungáricas 32(1-4): 3-48.

CAÑas Abril, P. 1978. Principales regiones físicogeográficas. 1: 3000 000. En: Atlas de Cuba. XX Aniversario del Triunfo de la Revolución Cubana. Instituto Cubano de Geodesia y Cartografía, La Habana. 44-45 pp.

Capote, R.P., N. Ricardo, D. Vilamajó, R. Oviedo \& E.E GARCía. 1987. Flora y Vegetación de la zona costera entre Daiquirí y Verraco, Parque Baconao, Santiago de Cuba. Acta Botánica Cubana 48: 1-28.

Cobiella, J.L. 1983. Algunas consideraciones sobre el origen de la Fosa de Bartlett. Revista Minería y Geología 1: 30-56.

Coleman, R.G. \& E.B. Alexander. 2004. Geologic setting of the ultramafic floristic districts of Cuba. In: R. S. Boyd, A. J. M. Baker \& J. Proctor (eds.), Rocas ultramáficas: sus suelos, vegetación y fauna. pp. 1-7. Science Reviews. UK.

Chiappy, C., P. Herrera \& L. Iñiguez. 1988. Aspectos botánicos y valores para la conservación de la naturaleza de la llanura costera del norte de la Provincia de Las Tunas, Cuba. Acta Botánica Cubana 61: 1-26.

Díaz, J. L., A.H. Portela, P. Blanco \& A. Magaz. 1986. Los principios básicos de la clasificación del relieve cubano. En: J. Díaz, A. H. Portela, P. Blanco \& A. Magaz (eds.), Los principios básicos de la clasificación morfoestructural del relieve cubano y su aplicación en la Región Centro-Oriental de Cuba. Instituto de Geografía, pp. 1-12. Academia de Ciencias de Cuba, La Habana.

Dietrich, H. 2000. Plantaginaceae. En: W. Greuter, H. Manitz \& R. Rankin Rodríguez (eds.). Flora de la República de Cuba. Serie A, Plantas Vasculares. Fascículo 5(7). Ed. Koeltz Scientific Books. Königstein. 12 pp.

Dressler, S. 2000. Marcgraviaceae. En: W. Greuter, H. Manitz \& R. Rankin Rodríguez (eds.). Flora de la República de Cuba. Serie A, Plantas Vasculares. Fascículo 5(4). Ed. Koeltz Scientific Books. Königstein. 14 pp. 
Gayana Bot. 66(2), 2009

FAgILdE EspinosA, M.C. 1998. El endemismo en NipeSagua-Baracoa. En: N. Viña Bayés, A. Fong G. $\&$ D. Maceira F. (eds.). Diversidad Biológica del Macizo Montañoso Nipe-Sagua-Baracoa. Tomo 2. pp. 598-608. Centro Oriental de Ecosistemas y Biodiversidad (BIOECO), Santiago de Cuba.

FAgilde Espinosa, M.C. 2000a. El endemismo en algunas formaciones vegetales arbóreas de Nipe-Sagua-Baracoa. Biodiversidad de Cuba Oriental 5: 41-44.

Fagilde Espinosa, M. C. 2000b. Fitogeografía. Fanerógamas endémicas. En: N. Viña Bayés, A. Fong G. \& D. Maceira F., (eds.). Diversidad Biológica del Macizo Montañoso Sierra Maestra. Tomo 1. pp. 427-435. Centro Oriental de Ecosistemas y Biodiversidad (BIOECO), Santiago de Cuba.

Fagilde Espinosa, M.C. 2001. El endemismo en las fanerógamas de Cuba Oriental.En: N. Viña Bayés, A. Fong G. \& D. Maceira F. (eds.). Diversidad Biológica de los Macizos Montañosos de Cuba Oriental. Tomo 1. pp. 503-511. Centro Oriental de Ecosistemas y Biodiversidad (BIOECO), Santiago de Cuba.

Fernández, M., A. López \& B. L. Toscano 1985. Las Rubiáceas endémicas de Cuba. En: Memorias del Primer Simposio de Botánica [2-5 julio], Tomo 1. pp. 91-98. La Habana.

Formell Cortina, F. 1989. Constitución geológica. En: Nuevo Atlas Nacional de Cuba. Instituto de Geografía de la Academia de Ciencias de Cuba e Instituto Cubano de Geodesia y Cartografía, La Habana. III.1.1.

Franco Flores, F., I. Castañeda Noa \& R. Oviedo Prieto. 2004. Flora ultramáfica de Motembo, Villa Clara, Cuba. En: R.S. Boyd, A.J.M. Baker \& J. Proctor (eds). Rocas ultramáficas: sus suelos, vegetación y fauna. pp. 65-71. Science Reviews. UK.

García, E.E., N. Ricardo, R. Oviedo \& R. Capote. 1985a. Flora y Vegetación del Área de Morrillo Chico, Santiago de Cuba. pp. 1-24. En: Memorias del Primer Simposio de Botánica [2-5 julio], Tomo 3. La Habana.

García, E.E., N. Ricardo, R. CApote, D. Vilamajó \& R. Oviedo. 1985b. Flora y Vegetación de la Sierra de la Gran Piedra, Santiago de Cuba. En: Memorias del Primer Simposio de Botánica [2-5 julio], Tomo 3. pp. 25-45. La Habana.

Gentry, A.H. 1982. Neotropical floristic diversity: phytogeographic connections between Central and South America. Pleistocens climatic fluctuations or an accident of the Andean orogeny. Annals of the Missouri Botanical Garden 69: 557-593.

Gentry, A.H. 1985. Contrasting phytogeographic patterns of upland and lowland Panamian plants. In: W.G. D'Arcy \& M. D. Correa (eds.), The Botany and natural history of Panama. Monograph Systematic Botany. Missouri Botanical Garden 10: 147-160.
Gentry, A. H. \& C.H. Dodson. 1987. Diversity and biogeography of neotropical vascular epiphytes. Annals of the Missouri Botanical Garden 74: 205-233

Gómez de la Maza, M. \& J.T. Roig y Mesa. 1914. Flora de Cuba (datos para su estudio). 2da parte. Comparación de la flora de Cuba con la de otros países. La Habana. p.182.

GonzÁlez Géigel, L. \& J. Bisse 1998. Linaceae. En: H. Manitz \& A. Gutjahr (eds.), Flora de la República de Cuba. Serie A, Plantas Vasculares. Fascículo 1(5). Ed. Koeltz Scientific Books. Königstein. 14 pp.

González Gutiérrez, P.A. \& J. Sierra Calzado. 2004. Aquifoliaceae. En: W. Greuter \& R. Rankin Rodríguez (eds.), Flora de la República de Cuba. Serie A, Plantas Vasculares. Fascículo 9(1). Ed. Gantner. Ruggell, Liechtenstein. 33 pp.

GonzÁlez-Torres, L.R., E.R., Bécquer Granados, R. Berazaín Iturralde \& J. Gutiérrez. 2004. Diversidad florística y vegetación del Tibisial: una localidad ultramáfica del macizo Guamuhaya, Cuba. En: R. S. Boyd, A. J. M. Baker, \& J. Proctor (eds.), Rocas ultramáficas: sus suelos, vegetación y fauna, pp. 73-78. Science Reviews. UK.

Greuter, W. 2002. Phytolaccaceae. En: W. Greuter, R. Rankin Rodríguez \& H. Manitz (eds.), Flora de la República de Cuba. Serie A, Plantas Vasculares. Fascículo 6(3). Ed. Koeltz Scientific Books. Königstein. 37 pp.

Gutiérrez Amaro, J. 2000. Flacourtiaceae. En: W. Greuter, H. Manitz \& R. Rankin Rodríguez (eds.), Flora de la República de Cuba. Serie A, Plantas Vasculares. Fascículo 5(1). Ed. Koeltz Scientific Books. Königstein. Pp. 76.

Gutiérrez Amaro, J. 2002. Sapotaceae. En: W. Greuter, R. Rankin Rodríguez \& H. Manitz (eds.), Flora de la República de Cuba. Serie A, Plantas Vasculares. Fascículo 6(4). Ed. Koeltz Scientific Books. Königstein. 59 pp.

HernándeZ, J.R. 1989. Geomorfología estructural del sistema montañoso de la Sierra Maestra y de las depresiones graben adyacentes. Editorial Academia. La Habana. 31 pp.

Hernández-Sarlabous, M. 1993. Análisis petrológico comparativo de las secuencias metavulcanógenas del arco insular mesozoico de las Antillas Mayores. Revista Minería y Geología 5(6): 24-31.

HernándeZ, M. \& Z. Canedo Salazar. 1995. Geoquímica de las ofiolitas meridionales de Cuba Oriental. Revista Minería y Geología 12(6): 32-38.

Iturralde-Vinent, M.A. 1982. Aspectos geológicos de la biogeografía. Ciencias Tierra Espacio 5: 85-100.

Hespenheide, H.A. 1973. A revision of the West Indian species of Lepanthes (Orchidaceae). Brittonia 25(3): 257-283.

HowarD, R.A. 1968. The ecology of an elfin forest in Puerto Rico 1. Introduction and composition 
Fitogeografía de las pluvisilvas de Cuba Oriental: Martínez-Quesada, E.

studies. Journal of Arnold Arboretum 49(4): 381-418.

ItURRALde-VInENT, M.A. 1988. Naturaleza geológica de Cuba. Editorial Científico-Técnica. La Habana. $146 \mathrm{pp}$.

ItURRALDE-Vinent, M.A. (ed.). 1996. Cuban ophiolites and volcanic arcs. The International Geological Correlation Programme. Project 364, Spect. Contr. 1. Miami, Florida.

IturRalde-Vinent, M.A. 1998 (2000). Sinopsis de la constitución geológica de Cuba. Acta Geológica Hispánica 33(1-4): 9-56.

Iturralde-Vinent, M.A. \& R.D.E. MacPhee. 1999. Paleogeographic of the Caribbean Region: implications for the Cenozoic biogeography. Bulletin of American Museum of Natural History 238: 1-95.

IturRalde-Vinent, M.A. (2002) 2004. Paleogeografía del Caribe 205-55 Ma. En: M.A. Iturralde-Vinent (ed.), Paleogeografía y biogeografía de Cuba y el Caribe. Primera Edición Digital. Museo Nacional de Historia Natural, La Habana.

Kuist, J. 1961. A revision of Dendrophthora (Loranthaceae). Wentia 6: 65-145.

Lastres, O. 1988. Dinámica de las reservas orgánicas y energéticas de la hojarasca en un bosque tropical semideciduo en Cuba. Tesis Dr. Ciencias Biológicas. Instituto de Ecología y Sistemática. 182 pp.

Leiva SÁnchez, A. T. 1992. Loranthaceae. En: Flora de la República de Cuba. Fontqueria 34: 5-16.

León, Hno. 1946. Flora de Cuba 1. Gimnospermas. Monocotiledóneas. Contribuciones Ocasionales Museo Historia Natural Colegio "De La Salle" 8, La Habana. 441 pp.

León, Hno. \& Hno. Alain 1951. Flora de Cuba 2. Dicotiledóneas: Casuarináceas a Meliáceas. Contribuciones Ocasionales Museo Historia Natural Colegio "De La Salle" 10, La Habana. $456 \mathrm{pp}$.

LóPez, A. 1998a. Algunas características del endemismo en la flora de Cuba Oriental. G. Halfter (comp.) La Diversidad Biológica en Iberoamérica II. Volumen Especial. Acta Zoológica Mexicana, n.s. pp. 47-82.

López Almirall, A. 1998b. Diversidad de la flora endémica en Cuba Oriental. Familias con endemismos distritales. Moscosoa 10: 136-163.

López Almirall, A., Toscano, B. L. \& M.M. Llerena. 1985. Las fanerógamas endémicas de Pinar del Río. En: Memorias del Primer Simposio de Botánica [2-5 julio], Tomo 1. pp. 53-90. La Habana.

López Almirall, A., M. Rodríguez Tasé \& CÁrdenas, A. 1993. El endemismo vegetal en MaisíGuantánamo (Cuba Oriental). Fontqueria 36: 399-420.

López Almirall, A., M. Rodríguez Tasé \& A. CÁrdenas. 1994a. El endemismo vegetal del Turquino (Cuba Oriental). Fontqueria 39: 395-431.

López Almirall, A., M. Rodríguez Tasé \& A. CÁrdenas. 1994b. El endemismo vegetal en Moa-ToaBaracoa (Cuba Oriental). Fontqueria 39: 433-473.

Magaz García, A.R. 1989. Hipsometría 1. 1: 1000000. En: Nuevo Atlas Nacional de Cuba. Instituto de Geografía de la Academia de Ciencias de Cuba e Instituto Cubano de Geodesia y Cartografía, La Habana. IV.1.2-3.

MAI, D. H. 2003. Styracaceae. En: W. Greuter \& R. Rankin Rodríguez (eds.), Flora de la República de Cuba. Serie A, Plantas Vasculares. Fascículo 7(2). Ed. Gantner. Ruggell, Liechtenstein. 9 pp.

MAI, D. 2005. Symplocaceae. En: W. Greuter \& R. Rankin Rodríguez (eds.), Flora de la República de Cuba. Serie A, Plantas Vasculares. Fascículo 10(9). Ed. Gantner. Ruggell, Liechtenstein. 20 pp.

Martínez Quesada, E. 1998. Relaciones florísticas de las fanerógamas no endémicas de Nipe-SaguaBaracoa. En: N. Viña Bayés, A. Fong G. y D. Maceira F. (eds.). Diversidad Biológica del Macizo Montañoso Nipe-Sagua-Baracoa. Tomo 2. pp. 609-612. Centro Oriental de Ecosistemas y Biodiversidad (BIOECO), Santiago de Cuba.

Martínez Quesada, E. 2000. Relaciones florísticas de las fanerógamas no endémicas de la Sierra Maestra. En: N. Viña Bayés, A. Fong G. y D. Maceira F. (eds.), Diversidad Biológica del Macizo Montañoso Sierra Maestra. Tomo 1. pp. 437-439. Centro Oriental de Ecosistemas y Biodiversidad (BIOECO), Santiago de Cuba.

Martínez Quesada, E. 2001. Relaciones florísticas de las fanerógamas no endémicas en la Región Oriental. En: N. Viña Bayés, A. Fong G. y D. Maceira F. (eds.), Diversidad Biológica de los Macizos Montañosos de Cuba Oriental. Tomo 1. pp. 512-516. Centro Oriental de Ecosistemas y Biodiversidad (BIOECO), Santiago de Cuba.

Mateo Rodríguez, J. \& M. Acevedo González. 1989. Regionalización Físico-Geográfica 5. 1: 3000 000. En: Nuevo Atlas Nacional de Cuba. La Habana. XII.2.1.

MÉndez SAntos, I.E. 2003. Verbenaceae. En: W. Greuter \& R. Rankin Rodríguez (eds.), Flora de la República de Cuba. Serie A, Plantas Vasculares. Fascículo 7(3). Ed. Gantner. Ruggell, Liechtenstein. $126 \mathrm{pp}$.

Montes, L., Oviedo, R. \& C. Chiappy. 1985. Aspectos florísticos generales de Pico Potrerillo, Topes de Collantes, Cuba. En: Memorias del Primer Simposio de Botánica [2-5 julio], pp. 100-130. Tomo 1. La Habana.

Moya López, C. \& A.T. Leiva SÁnchez. 2000. Checklist of the Palms of Cuba, with notes on their Ecology, Distribution and Conservation. Palms 44(2): 69-84.

NúÑEz JimÉnEZ, A. 1954. Geografía de Cuba. Editorial Lex, La Habana. 411 pp.

NúÑez Jiménez, A. \& N. ViÑa Bayés. 1989. Regiones Naturales-Antrópicas 6. 1:3000 000. En: Nuevo Atlas Nacional de Cuba, La Habana. XII.2.1.

Odreman, O. \& F. Ricardi. 1990. Flora paleozoica de la 
Región de Carache, Estado Trujillo, Venezuela. Parte I. Pecopteridea. En: Resúmenes. V Congreso Latinoamericano de Botánica (24-29 junio). pp. 274. La Habana.

Ortega, P. \& M. Arcia 1982. Determinación de las lluvias en Cuba durante la glaciación de Wisconsin mediante relictos edáficos. Ciencias Tierra Espacio 4: 85-104.

Oro Alfonso, J. R. 1989. Evolución paleogeológica 2. 1: 4000 000. En: Nuevo Atlas Nacional de Cuba. Instituto de Geografía de la Academia de Ciencias de Cuba e Instituto Cubano de Geodesia y Cartografía, La Habana. III. 1.4.

Panfet Valdés, C. 1998. Droseraceae. En: H. Manitz \& A. Gutjahr (eds.), Flora de la República de Cuba. Serie A, Plantas Vasculares. Fascículo 1(4). Ed. Koeltz Scientific Books. Königstein. 20 pp.

Panfet Valdés, C. 2005. Myrsinaceae. En: W. Greuter \& R. Rankin Rodríguez (eds.), Flora de la República de Cuba. Serie A, Plantas Vasculares. Fascículo 10(7). Ed. Gantner. Ruggell, Liechtenstein. 44 pp.

PÉREZ, J. 2005. Dilleniaceae. En: W. Greuter \& R. Rankin Rodríguez (eds.), Flora de la República de Cuba Serie A, Plantas Vasculares. Fascículo 10(3). Ed. Gantner. Ruggell, Liechtenstein. 25 pp.

Portela Peraza, A. H., J. L. Díaz Díaz, J. R. Hernández Santana, A. R. Magaz García \& P. Blanco SEGUNDO. 1989. Geomorfología 11. 1: 1000000. En: Nuevo Atlas Nacional de Cuba. Instituto de Geografía de la Academia de Ciencias de Cuba e Instituto Cubano de Geodesia y Cartografía, La Habana. IV.3.2-3.

RANKIN RodríGUEZ, R. 1998. Aristolochiaceae. En: H. Manitz \& A. Gutjahr (eds.), Flora de la República de Cuba. Serie A, Plantas Vasculares. Fascículo 1(2). Ed. Koeltz Scientific Books. Königstein. 39 pp.

Rankin Rodríguez, R. 2003. Polygalaceae. En: W. Greuter \& R. Rankin Rodríguez (eds.), Flora de la República de Cuba. Serie A, Plantas Vasculares. Fascículo 7(1). Ed. Gantner. Ruggell, Liechtenstein. $52 \mathrm{pp}$.

RANKIN Rodríguez, R. 2005. Cleomaceae. En: W. Greuter \& R. Rankin Rodríguez (eds.), Flora de la República de Cuba Serie A. Plantas Vasculares. Fascículo 10(1). Ed. Gantner. Ruggell, Liechtenstein. 24 pp.

RAVEN, P. \& D. AXELROD. 1974. Angiosperm biogeography and past continental movements. Annals of the Missouri Botanical Garden 61: 539-673.

Reyes, O.J. 1994. Algunas consideraciones sobre la biodiversidad cubana, con énfasis en la flora fanerógama, En: Memorias Conferencias Cuba-México, ENCB del IPN. pp. 102-129. México, D.F.

Reyes, O.J. 2006. Clasificación de la vegetación de la Sierra Maestra. Biodiversidad de Cuba Oriental 8: $28-42$.

Reyes, O.J., K. Mustelier, \& D. Reyes. 1991. Distribución mundial y vías de migración de las hepáticas cubanas. En: M.C. Delgadillo (ed.), Memorias del II Simposio Latinoamericano de Briología (La Habana, Junio de 1990). pp. 1-10. Instituto de Biología, UNAM, México, D. F.

Reyes, O.J. \& E. Del Risco. 1994. El endemismo de la flora fanerógama de los pinares del Noreste de Cuba Oriental. En: Memorias Conferencias Cuba-México, pp. 141-151. ENCB del IPN, México, D. F.

Reyes, O.J., R. Cruz Doimeadiós \& M. GonzÁlez. 2000. Relaciones fitogeográficas de la flora fanerógama de las cuencas de los ríos Toa y Duaba, Cuba. Biodiversidad de Cuba Oriental 5: 58-64.

Reyes, O.J. \& F. Acosta Cantillo. 2005a. Cuba: Parque Nacional "Alejandro de Humboldt" (Vegetación). En: A. Fong G., D. Maceira F., W.S. Alverson \& T. Wachter (eds.), Rapid Biological Inventories, Report 14. The Field Museum, Chicago. 370 pp.

Reyes, O.J. \& F. Acosta. 2005b. Cuba: Parque Nacional La Bayamesa (Vegetación). En: F.D. Maceira, A. Fong G., W.S. Alverson \& T. Wachter (eds.), Rapid Biological Inventories, Report 13 The Field Museum, Chicago. 243 pp.

Ricardo, N., D. Vilamajó, R. Oviedo, E.E. García \& J. BASTART. 1985. La vegetación de la Meseta de Santa María del Loreto, Santiago de Cuba. En: Memorias del Primer Simposio de Botánica [2-5 julio], Tomo 3. pp. 46-58. La Habana.

Robinson, E. 1976. Minerals and plate tectonics in Jamaica. Jamaica Journal. 10: 86-92.

Robinson, E., J.F. Lewis \& R.V. CANT. 1977. Field guide to aspects of the geology of Jamaica. In: P.H. Mattson (ed.), West Indies island arcs (Benchmark Papers in Geology 33). Dowden, Hutchinson \& Ross Inc., Stroudsburg, Pennsylvania, 210-221.

Rodríguez Fuentes, A. 2000. Elaeocarpaceae. En: W. Greuter, H. Manitz \& R. Rankin Rodríguez (eds.), Flora de la República de Cuba. Serie A, Plantas Vasculares. Fascículo 3(3). Ed. Koeltz Scientific Books. Königstein. 12 pp.

Rosen, D.E. 1985. Geological hierarchies and biogeographic congruence in the Caribbean. Annals of the Missouri Botanical Garden 72: 636-659.

Roughgarden, J. 1995. Anolis Lizards of the Caribbean. Ecology, Evolution and Plate Tectonics. Oxford University Press, London. 200 pp.

SameK, V. 1973. Regiones fitogeográficas de Cuba. Academia de Ciencias de Cuba. Serie Forestal 15: $1-60$.

Saralegui Boza, H. 2000. Chloranthaceae. En: W. Greuter, H. Manitz \& R. Rankin Rodríguez (eds.), Flora de la República de Cuba. Serie A, Plantas Vasculares. Fascículo 3(2). Ed. Koeltz Scientific Books. Königstein. 12 pp.

Saralegui Boza, H. 2004. Piperaceae. En: W. Greuter \& R. Rankin Rodríguez (eds.), Flora de la República de Cuba. Serie A, Plantas Vasculares. Fascículo 9(3). Ed. Gantner. Ruggell, Liechtenstein. 94 pp. 
Fitogeografía de las pluvisilvas de Cuba Oriental: Martínez-Quesada, E.

Schmid, G. 1969. Vegetationsgeographie auf ökologisch-soziologischer Grundlage. Teubner Verlgsgesellschaft, Deutsche Demokratische Republik. 575 pp.

SeIfriz, W. 1943. The plant life of Cuba. Ecology Monograph 13: 375-426.

Sierra Calzado, J. 2000. Begoniaceae. En: W. Greuter, H. Manitz, R. Rankin Rodríguez (eds.), Flora de la República de Cuba. Serie A, Plantas Vasculares. Fascículo 3(1). Ed. Koeltz Scientific Books. Königstein. 27 pp.

Thiv, M. 2002. Gentianaceae. En: W. Greuter, R. Rankin Rodríguez \& H. Manitz (eds.), Flora de la República de Cuba. Serie A, Plantas Vasculares.
Fascículo 6(1). Ed. Koeltz Scientific Books. Königstein. $40 \mathrm{pp}$.

Urquiola Cruz, A. J., J. R. Aguilar Trujillo \& M. Betancourt Gandul. 2000. Mayacaceae. En: W. Greuter, H. Manitz \& R. Rankin Rodríguez (eds.), Flora de la República de Cuba. Serie A, Plantas Vasculares. Fascículo 5(5). Ed. Koeltz Scientific Books. Königstein. 8 pp.

Viña BAYÉs, N. 2005. Cuba: Parque Nacional La Bayamesa (Características FísicoGeográficas). En: D. Maceira F., A. Fong G., W.S. Alverson \& T. Wachter (eds.), Rapid Biological Inventories, Report 13. The Field Museum, Chicago. 243 pp.

Recibido: 07.04.09

Aceptado: 07.08.09 